\title{
1 Consolidated sediment resuspension in model vegetated canopies
}

2

3 Jordi Colomer $^{1}$, Aleix Contreras ${ }^{1}$ and Andrew Folkard ${ }^{2}$ and Teresa Serra ${ }^{1}$

4

$5 \quad{ }^{1}$ Department of Physics,

6 Campus Montilivi, Escola Politècnica Superior II

7 University of Girona

817071 Girona (Spain)

9

10

${ }^{2}$ Lancaster Environment Centre, Lancaster LA1 4YQ

11 United Kingdom

12

Corresponding author: teresa.serra@udg.edu

Running title: Consolidated bed resuspension in canopies

Keywords: oscillating grid, isotropic turbulence, sediment re-suspension, turbulent kinetic energy, submerged vegetation. Acknowledgments

This research was funded by the University of Girona, through the grant MPCUdG2016-006 and by the Ministerio de Economía, Industria y Competitividad of the Spanish Government through the grant CGL2017-86515-P. 


\section{Abstract}

Aquatic plants, turbulence and sediment fluxes interact with each other in a complex, nonlinear fashion. While most studies have considered turbulence as being generated primarily by mean flow, it can, however, also be generated by the action of the wind or by the night cooling convection at the surface of the water column. Here, we study turbulent interaction with vegetation and the effects it has on sediment suspension, in the absence of mean flow. In a water tank containing a base layer of sediment, turbulence was generated by oscillating a grid with the main objective being to determine the differences in sediment resuspension in sediment beds over a wide range of consolidation times (1h-3days), for a set of model canopies with different structural characteristics: density and flexibility, and for three types of sediment beds. The greater the consolidation time was, the lower the sediment resuspension. For bed consolidation times below six hours, the concentration of resuspended sediment was approximately constant and had no dependence on turbulence intensity. However, for higher bed consolidation times, between six and three days, the resuspension of the sediment beds increased with turbulence intensity (defined in terms of turbulent kinetic energy; TKE hereafter). The TKE within the sparse flexible canopies was higher than that in the sparse rigid canopies, while within the dense flexible canopies it was below that of the rigid canopies. Therefore, the sediment resuspension in the sparse flexible canopies was greater than that of the sparse rigid canopies. In contrast, the sediment resuspension in the dense flexible canopies was lower than that of the dense rigid canopies. Using different sediment types, the results of the study indicate that sediments with greater concentrations of small particles (muddy beds) have higher concentrations of resuspended sediment than sediment beds that are composed of larger particle sizes (sandy beds). 


\section{List of symbols and abbreviations}

62

63

64

$65 \quad b$

$66 \mathrm{C}$

$67 \mathrm{C}_{\mathrm{t}}$

Total area studied $\left(\mathrm{cm}^{2}\right)$

$68 \mathrm{C}_{0}$

Acoustic Doppler Velocimeter

69 CSS

Plant width (mm)

$70 \mathrm{~d}$

Suspended sediment concentration $\left(\mu \mathrm{g} \cdot \mathrm{L}^{-1}\right)$

$71 \mathrm{E}$

Suspended sediment concentration with time $\left(\mu \mathrm{g} \cdot \mathrm{L}^{-1}\right)$

$72 \mathrm{f}$

Initial suspended sediment concentration, at $\mathrm{t}=0 \mathrm{~s}\left(\mu \mathrm{g} \cdot \mathrm{L}^{-1}\right)$

$73 \mathrm{~h}_{\mathrm{w}}$

Relative suspended sediment concentration in the steady state $\left(\mu \mathrm{g} \cdot \mathrm{L}^{-1}\right)$

$74 \mathrm{~h}_{\mathrm{S}}$

Diameter of the plant model (mm)

$75 \mathrm{k}$

Modulus of elasticity $(\mathrm{Pa})$

Grid oscillation frequency $\left(\mathrm{s}^{-1}\right)$

Mean water depth (m)

Length of the rigid canopy model (m)

$76 \quad \mathrm{k}_{0}$

Turbulent kinetic energy

$77 \quad 1$

Turbulent kinetic energy profile at the boundary

Integral length scale ( $\mathrm{mm}$ )

$79 \mathrm{n}$

Spacing between bars in oscillating grid (m)

80 OGT

Number of plants per square meter

81 PVC

Oscillating Grid Turbulence

$82 \mathrm{R}^{2}$

Polyvinyl chloride

$83 \mathrm{~s}$

Correlation

$84 \quad \mathrm{SFV}$

Stroke (m)

85 SPF

Submerged Flexible Vegetation

86 SRV

Solid Plant Fraction (\%)

$87 \quad \mathrm{t}$

Submerged Rigid Vegetation

88 TKE

Time (s)

89 TSS

Turbulent Kinetic Energy $\left(\mathrm{m}^{2} \cdot \mathrm{s}^{-2}\right)$

$90 \mathrm{u}, \mathrm{v}, \mathrm{w}$

Total Suspended Sediment $\left(\mathrm{g} \cdot \mathrm{L}^{-1}\right)$

$91 \mathrm{U}$

Components of the Eulerian velocity

$92 \mathrm{u}$

Time averaged velocity $\left(\mathrm{m} \cdot \mathrm{s}^{-1}\right)$

93 WP

Turbulent component of velocity $\left(\mathrm{m} \cdot \mathrm{s}^{-1}\right)$

Without plants 


$\begin{array}{lll}94 & \mathrm{Z} & \text { Vertical direction } \\ 95 & \mathrm{Z}_{0} & \text { Distance from the grid to the water surface }(\mathrm{m}) \\ 96 & \lambda_{1} & \text { Lambda parameter } 1 \\ 97 & \lambda_{2} & \text { Lambda parameter } 2 \\ 98 & \rho_{\omega} & \text { Water density }\left(\mathrm{kg} \cdot \mathrm{m}^{-3}\right) \\ 99 & \rho_{\mathrm{v}} & \text { Plant density }\left(\mathrm{kg} \cdot \mathrm{m}^{-3}\right) \\ 100 & v & \text { Kinematic viscosity }\left(\mathrm{m}^{2} \cdot \mathrm{s}^{-1}\right) \\ 101 & & \end{array}$




\section{Introduction}

Along coastal and littoral lake zones, submerged aquatic vegetation affects ambient hydrodynamics by reducing water column turbulence, leading to a reduction in sediment erosion, and thus increasing the water column clarity in lakes and saltmarshes [1-3]. When the water clarity is enhanced, there is greater light penetration and this creates positive feedback for the canopy [4-7].

Sediment resuspension and turbidity variations have been observed to impact plant development and hydrodynamics. For example, the construction of a large dam caused the ecosystem in the Dutch Wadden Sea to collapse from a vegetated to a bare state as a result of the increase in turbidity [8]. This then led to eutrophication, caused by a decrease in light availability, and the migration of seagrass meadows to shallower waters [7]. In Lake Taihu, Zhu et al. [9] found that under similar wind speeds, the presence of macrophytes reduced sediment resuspension rates by 29 -fold. Consequently, eutrophication and cyanobacteria blooms along the calm shoreline areas of Lake Taihu negatively impact on its ecosystem [10]. Comparative data in the Mediterranean show that a canopy of Posidonia oceanica may reduce resuspension rates by three- to seven-fold compared to those in the adjacent unvegetated floor $[11,12]$.

Plants with different morphologies may alter the hydrodynamics differently and, therefore, the processes of erosion, suspension and deposition [1, 3, 13-15]. Wu et al [10] found that the zones covered by littoral aquatic macrophytes in Lake Taihu had thicker sediment layers. The amount of sediment erosion and resuspension is known to be governed by the intensity of the external forcing event [16] and canopy properties [17]. The sediment resuspension by unidirectional flow through a simulated canopy has been found to be a function of both the flow velocity and the wakes produced by the stem scale turbulence [18]. Therefore, a threshold in the shear stress can be stablished as a function of the flow velocity and the array of the cylinders. In contrast, field studies have evidenced the role between the sediment resuspension and the presence of intermittent turbulent events [19]. Studies using emergent plants have shown that turbulence inside canopies decreases linearly with increasing stem density, and that even low densities of plants can produce substantial reductions in turbulence [20]. On the other hand, Bouma et al [21] found that sparse canopies of rigid plants increased flow velocity, and thus sediment scouring and resuspension. The high flow velocities in sparse canopies can also impact on the distribution of seeds, nutrients and sediments [22, 23]. 
A great deal of research has been carried out to determine the effects emergent and submerged vegetation have on hydrodynamics $[13,14,24-27]$. Turbulence is generated in the wake of individual stems as well as in the canopy as a whole, and also by shear as a result of the velocity gradients in the mean flow field [28]. Density and plant flexibility are the key parameters that control the TKE attenuation within canopies and therefore the sediment resuspension [15]. However, most of the work has been carried out in flows dominated by waves or mean currents and not in cases where the turbulence is the main hydrodynamic force. The littoral zones of lakes and ponds are regions with limited advection and the main source of turbulence comes from wind action on the surface, or night convection [29]. In these systems, the turbulence produced at the water surface decreases with depth. Therefore, further work needs to be done to quantify the effect that both flexibility and canopy density have on the sediment resuspension produced by zero-mean flow turbulence. One way of approaching this problem is by running experiments using an oscillating grid device. Oscillating grids produce nearly isotropic zeromean flow turbulence [30-32] and have been used since the 1990s to study isotropic turbulence in the absence of the mean shear associated with flowing water. The properties of the turbulence are determined by the geometry of the grid, the frequency and amplitude of the oscillations, and the distance from the grid [33,34]. Oscillating grid turbulence devices (OGT) can be used as an analogue to open-channel flow systems by setting the operational parameters of the grid (stroke, frequency, etc.) such that the total kinetic energy of the turbulence matches that expected either at the bed or at the free surface for an open-channel flow [35].

OGTs are used to produce controlled turbulent fields allowing turbulence in physical phenomena to be understood. OGTs have been used to study the resuspension of both cohesive [36] and non-cohesive [37] sediments. Tsai and Lick [36] found that the concentration of resuspended cohesive sediment was proportional to the oscillation frequency of the grid. Huppert et al [37] found that above a critical oscillating frequency, a given mass of noncohesive sediment particles can be kept in suspension indefinitely. This critical frequency depends on the diameter of the sediment particles. Orlins and Gulliver [35] used OGTs to study sediment resuspension from bare beds with two different consolidation times ( 2 and 11 days). For the same level of TKE, less-consolidated sediment beds are subject to greater amounts of resuspension. Given than turbulence can act on sediment beds on short time scales, this study also quantifies the effects turbulence has on beds from short (hours) to long consolidation times (days), therefore covering a greater range of consolidation times than that considered by Orlins and Gulliver [35] In canopies of aquatic vegetation, the turbulence induced by the wind affects 
the bottom boundary layer of the flow field in a manner that depends on the canopies' properties and the bed's degree of consolidation [38]. In addition, this study investigates the induced resuspension of natural cohesive partially consolidated sediment beds by turbulence in nonvegetated and vegetated environments under zero-mean flow turbulence. In this case, the entrainment of sediment particles from the interface is a result of turbulent fluctuations rather than the presence of a mean flow [39]. For this reason, an OGT has been considered suitable for studying the sediment resuspension. The canopy properties, such as the plant flexibility and canopy density, are expected to play an important role in the attenuation of pure isotropic turbulence, which has not been previously determined. Therefore, different canopy densities and plant models composed of flexible, rigid and semi-rigid plants will be considered. Furthermore, the sediment characteristics will also be explored. For this purpose, three sediments with different particle distributions will be used for the experiments.

\section{Methodology}

\subsection{Experimental setup}

The study was conducted in an oscillating grid turbulence chamber (Fig. 1) consisting of a box made of Plexiglas ${ }^{\circledR}$ whose interior dimensions measured $0.28 \mathrm{~m} \times 0.28 \mathrm{~m} \times 0.33 \mathrm{~m}$. This was filled with water to a depth, $h_{w}$, of $0.315 \mathrm{~m}$. A Plexiglas® grid was suspended from above the chamber such that its center was $z_{0}=0.065 \mathrm{~m}$ below the water surface $(0.25 \mathrm{~m}$ above the bottom of the chamber). The oscillating grid was constructed with $1 \mathrm{~cm}$ wide and thick Plexiglas ${ }^{\circledR}$ square bars. Following the same technical requirements like those of De Silva and Fernando [30], the grid was composed of $5 \times 5$ bars, with $M=0.05 \mathrm{~m}$ spacing (or 'mesh size') between the bars giving it a $31 \%$ solidity (defined as the fractional solid area occupied by bars). Using a variable speed motor located outside the tank, with a fixed stroke $s=0.05 \mathrm{~m}$, and frequencies $f=2.8,3.3,3.8,4.3$ and $4.8 \mathrm{~Hz}$, the grid was oriented horizontally and oscillated vertically. A clearance of $2 \mathrm{~mm}$ between the sidewalls and the grid was maintained. We defined the vertical direction as $z$ (positive downwards), and $z=0 \mathrm{~cm}$ as the mean vertical position of the oscillating grid.

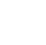




\subsection{Vegetation models}

Simulated canopies of either rigid, semi-rigid or flexible vegetation were placed in the tank prior to each experimental run. The rigid canopy models consisted of $d=6 \mathrm{~mm}$ wide and $h_{s}=0.10 \mathrm{~m}$ long PVC cylinders (Fig. 2a). The flexible canopy models were constructed by taping flexible polyethylene blades to rigid PVC dowels $0.02 \mathrm{~m}$ long and $6 \mathrm{~mm}$ in diameter (Fig. 2b). Each simulated plant had eight $4 \mathrm{~mm}$ wide, $0.10 \mathrm{~m}$ long and $0.07 \mathrm{~mm}$ thick plastic blades. These flexible plant simulants were dynamically and geometrically similar to typical seagrasses, as described by Ghisalberti and Nepf [40], Folkard [41], Pujol et al [13] and El Allaoui et al [42]. The ratio between the thickness and the height of the plant was $7 \times 10^{-4}$, similar to that used by Folkard [41] of $8 \times 10^{-4}$. The aspect ratio of the plant (ratio between the width of the leaves and its height) was 0.04, the same as that used by Folkard [41] who used $0.25 \mathrm{~m}$ long and $0.01 \mathrm{~m}$ wide leaves. Therefore, the flexible plant model simulates the behavior of a Posidonia oceanica canopy under a turbulent flow. Blade density was less than that of water (as is the case for real seagrasses) so that, at rest, the flexible canopy height was the same as that of the rigid canopy. The semi-rigid canopy was made of nylon threads each $2 \mathrm{~mm}$ in diameter (Fig 2c). To compare semi-rigid to flexible vegetation at $d=6 \mathrm{~mm}$, eight nylon threads were stacked together at the base to mimic the equivalent number of blades (Fig. 2c) to those used for flexible plants.

Following Pujol et al [3], the canopy density was varied and quantified between runs using the solid plant fraction $S P F=100 n \pi(d / 2)^{2} / A$, where $n$ is the number of plant stems, and $A$ is the total bed surface area covered by the canopy. For the flexible canopies, $d$ was taken as the diameter of the rigid dowels at the base of the plant $(6 \mathrm{~mm}) . S P F \mathrm{~s}$ of $1,2.5,5,7.5$ and $10 \%$ were used for the rigid canopy runs, SPFs of 2.5, 5, 7.5 and $10 \%$ for the flexible runs and an SPF of $2.5 \%$ was used for the semi-rigid canopy (Table 1, Fig. 2c-h). These SPFs corresponded to densities $N$ of $354,884,1768,2652$ and 3536 plants $\cdot \mathrm{m}^{-2}$, which is in line with the medium to dense seagrass densities found in the field [12,43-45]. To create each canopy, the plants were secured into $6 \mathrm{~mm}$-diameter holes, which were arranged into a regular grid with $0.01 \mathrm{~m}$ center-to-center spacing on a plastic base board. The position of each plant on this grid was made using a random number generator $[13,46]$. Holes left unfilled once all the plants had been positioned were covered with tape to eliminate any potential effect the hole may have had. 
In addition, the vertical variation in canopy density varied from rigid to semi-rigid and to flexible canopies. Following Neumeier and Amos [47], the vertical variation in the canopy density was assessed from the lateral obstruction of the canopy by taking a lateral picture of a $2.5 \mathrm{~cm}$ thick canopy in front of a white background. Semi-rigid and flexible blades were painted black to increase the contrast in the image. Images of the lateral obstruction were digitized, and image analysis techniques were applied to differentiate the vegetation from the background. Finally, the lateral obstruction percentage was calculated. While rigid canopies had a lateral obstruction that remained constant with height, the lateral obstruction of the flexible plants varied with height and maximum percentages being from $\mathrm{z}=18 \mathrm{~cm}$ to $\mathrm{z}=22 \mathrm{~cm}$ (Fig. 3). The flexible $10 \%$ SPF canopies reached greater lateral obstruction areas (of 33\%) than the rigid canopies (of 16\%). For the semi-rigid canopy of $2.5 \%$ SPF, the maximum lateral obstruction area of the canopy was of $6.7 \%$, i.e., midway between that of the rigid and flexible canopies.

\subsection{Sediment bed emplacement}

Once the simulated canopy had been secured at the base of the experimental tank, and the tank had been filled with water, the bottom of the tank was then covered with sediment. Three types of sediment of different compositions were used (Table 1). Enough sediment from the marsh and lake areas was obtained in situ to perform all the experiments according to the designed experimental conditions. The sediment was cleaned to remove leaves and roots, dried and then sieved to remove particles larger than $500 \mu \mathrm{m}$.

The sediment particle size distribution (i.e. the sediment concentration $C$ versus its particle size diameter $d$ )for each sediment type used was analyzed with the Lisst-100X, (Sequoia Scientific, Inc., WA, USA) a laser particle size analyzer which has been used extensively and found to be appropriate for measuring either organic [48] or inorganic particles [12, 49]. Based on the classification from Rijn [50] and Blott and Pye [51], the sediment was divided into three ranges of particle diameter (Fig. 4). The first $(2.5-6.0 \mu \mathrm{m})$ corresponds to very fine silts (strongly cohesive), the second $(6.0-170 \mu \mathrm{m})$ to fine to coarse silts and small sand particles (weakly cohesive), and the third $(>170 \mu \mathrm{m})$ to small and medium sand particles. Considering the particle number distribution, the sediment analysis showed that $\approx 98 \%$ of the particles fell within the first range, while particles within the second range accounted for the remaining $2 \%$. However, in considering the particle volume concentration for the three sediment types, 
particles in the first range accounted for $38.2 \%$ (marsh), $29.73 \%$ (lake) and $24.6 \%$ (synthetic) of the total concentration. An increase in the percentage of small particles in the sediment distribution is expected to increase the cohesive properties of the sediment.

For the case without plants, experiments with different sediment bed thicknesses were considered to determine the effect this would have on the results obtained. The bottom of the tank was covered with a sediment layer to the uniform heights of $3.8 \mathrm{~mm}, 2.5 \mathrm{~mm}$ and $1.3 \mathrm{~mm}$, which corresponded to dry mass concentrations of $300 \mathrm{gL}^{-1}, 200 \mathrm{gL}^{-1}$ and $100 \mathrm{gL}^{-1}$, respectively. This seeding was performed by manually moving a tube (connected to the container) holding the homogeneous sediment mixture around the bottom of the chamber through the vegetation. The seeding resulted in a cloud of particles $\approx 1 \mathrm{~cm}$ in height, which was, following Ros et al [15], then left to settle. Figure 5 shows the concentration corresponding to the resuspended bottom sediment particles versus the TKE for the three sediment layers. The greater the sediment height at the bottom was, the higher the concentration of resuspended particles. Scouring was not observed in any of experiments that had the $3.8 \mathrm{~mm}$ and $2.5 \mathrm{~mm}$ high beds. All experiments were initiated with a consolidated bottom bed height of $2.5 \mathrm{~mm}$.

Once the sediment was resuspended, the particle volume distribution of the sediment for the second and third particle range was approximately constant throughout all the experiments for the three sediment types. For this reason, these larger particles were not considered in the analysis, and only particles in the smallest size range i.e., the strongly cohesive range, were analyzed.

\subsection{Turbulence measurements and analysis}

The three-dimensional turbulent velocity field $(u, v, w)$ inside the tank was measured with a three-component Acoustic Doppler Velocimeter ( $A D V$ ) (Sontek/YSI16-MHzMicroADV). The $A D V$ has an acoustic frequency of $16 \mathrm{MHz}$, a sampling volume of $90 \mathrm{~mm}^{3}$, a sampling frequency of $50 \mathrm{~Hz}$ and measures in the range $0-30 \mathrm{~cm} \mathrm{~s}^{-1}$. The distance between the head of the $A D V$ and the sampling volume was $0.05 \mathrm{~m}$. The $A D V$ was mounted onto a movable vertical frame allowing it to be manually situated at working depths between $z=0.10 \mathrm{~m}$ and $z=0.24 \mathrm{~m}$. For all experiments, the $A D V$ was placed horizontally $0.07 \mathrm{~m}(1.4 \times$ the mesh size $)$ from one side wall and $0.12 \mathrm{~m}(2.4 \times$ the mesh size $)$ from the other side wall to avoid side-wall effects, 
as suggested by Orlins and Gulliver [35]. In addition, following De Silva and Fernando [30], the mesh endings were designed to reduce mean secondary circulation. To avoid any spikes in the data coming from artifacts of instrument operation rather than being representative of the flow, $A D V$ measurements with beam correlations below $70 \%$ and signal to noise ratio (SNR) above in the range $15-30 \mathrm{~dB}$. Spikes and spurious data were discarded using the method by Goring and Nikora [52]. The use of single point $A D V$ measurements for characterizing $O G T$ can be justified by noting that several authors $[30,53,54]$ found that at a certain distance from the grid, turbulence is isotropic and the velocity fluctuations $u^{\prime}, v^{\prime}$ and $w^{\prime}$ are proportional to $1 / z$. It seems, therefore, plausible to use single-point $A D V$ measurements in this context, at least at $|z|>3 M$, where $M$ is the spacing between bars[55]. In the present study, $M=5 \mathrm{~cm}$, therefore for $|z|>15 \mathrm{~cm}$, the turbulence is expected to be isotropic. Furthermore, for the rigid vegetation with $S P F=1 \%$ and $2.5 \%$, in order to test for the horizontal homogeneity of the turbulence field, vertical velocity profiles with the $A D V$ were carried out at eight different horizontal locations. Maximum differences of $4 \%$ between the TKE measured at different positions were obtained. the Reynolds stresses at each location were calculated and no differences were obtained between locations when considering the margin of error (data not shown). Additional tests were made to guarantee the horizontal homogeneity. The exuberance, i.e. the ratio of upward $\left(u^{\prime} w^{\prime} \geq 0\right)$ to downward $\left(u^{\prime} w^{\prime} \leq 0\right)$ fluxes of momentum, was calculated following Rotach [56]. The exuberance was close to -1 , indicating that there was equal contribution of downward to upward flux of momentum. Consequently, single point $A D V$ measurements were used thereafter.

To obtain valid data acquisition within the canopy for the densest canopies of flexible plants and in accordance with Neumeier and Ciavola [57], Pujol et al [3] and Pujol et al [13], a few stems were removed (a maximum of 3 stems for the $S P F=10 \%$ canopy density) to avoid blocking the pathway of the $A D V$ beams. To minimize the effect this 'hole' has only a few stems were repositioned. For the dense flexible canopies, a thin ( $0.5 \mathrm{~mm}$ thick) $4 \mathrm{~cm}$-wide ring was situated $1 \mathrm{~cm}$ above the $A D V$ sensors to avoid them being blocked by the flexible plants. This metal ring was fixed with two stems of the same material that were attached to the dowels of the plants. Measurements of the flow velocities for the $S P F=0 \%$ experiments were taken with and without the ring and no differences were observed.

For each experiment, a vertical velocity profile was taken from a $z=0.10 \mathrm{~m}$ to $z=0.24 \mathrm{~m}$ depth (see Fig. 1) at $0.01 \mathrm{~m}$ intervals to obtain the turbulence field. Thus, the vertical profiles covered 
measurements inside and above the canopy. At each depth, the instantaneous water velocity $(u$, $v, w)$ was measured for 10 minutes (i.e. 30,000 measurements for each velocity component) and then decomposed as $u=U+u^{\prime}$, where $\mathrm{U}$ is the time-averaged velocity component in one horizontal direction $(x)$ and $u^{\prime}$ is the turbulent component in this direction. The velocity components $v$ (speed in the y-direction - the horizontal direction orthogonal to the $\mathrm{x}$-direction) and $w$ (speed in the vertical direction) were similarly decomposed into $V+v^{\prime}$ and $W+w^{\prime}$, respectively. The turbulent kinetic energy per unit mass (TKE) was then calculated from the mean of the square values of the three turbulent components:

$T K E=\frac{1}{2}\left(\overline{u^{\prime 2}}+\overline{v^{\prime 2}}+\overline{w^{\prime 2}}\right)$

One of the characteristics of the zero-mean shear flow in the OGT device is that there is no recirculation in the system, i.e. the mean velocities are zero. Since the effect of the canopy is not known, the total kinetic energy $\left(K E=\frac{1}{2}\left(U^{2}+V^{2}+W^{2}\right)\right)$ can be a parameter to check for the presence of zero mean currents (Fig. 6a and b). Results show that in all cases, and considering the error margin, the $K E$ remains below the $A D V$ noise. The other characteristic of the zero-mean shear in the OGT is that the TKE decreases with $z^{-2}$ for the region of homogeneous turbulence [55]. In the present study, all experiments with and without plants presented a linear relationship between TKE and $z^{-2}$ for $z>15 \mathrm{~cm}$ (Fig. 6c), i.e. $z>3 M$ in the homogeneous turbulent zone.

\subsection{Sediment entrainment measurements}

The downward diffusion of grid-generated turbulence was able to erode the sediment bed and maintain a sediment load in the water column as momentum was transferred to the sediment. Within the column, sediment samples of $80 \mathrm{~mL}$ were obtained using a pipette introduced through the opening of the lid situated on top of the experimental tank. Samples were collected from two different depths $(z=0.1 \mathrm{~m}$ i.e. $0.05 \mathrm{~m}$ above the canopy, and $z=0.22 \mathrm{~m}$ i.e. $0.03 \mathrm{~m}$ above the bottom). For all the experimental runs, the particle volume distribution of suspended sediment was measured using the Lisst-100X laser particle size analyzer. From these measurements, the particle volume concentration in each range (Fig. 4) was obtained as the sum of the particle volume concentration of all the particles within the size range. 
Given that the smaller particles in the size spectra can remain in suspension quasi-indefinitely, suspended sediment concentration $(C)$ was calculated relatively, as the value measured at a time $t\left(C_{t}\right)$ subtracted from the value measured prior to the start of the oscillations at $t=0\left(\mathrm{C}_{0}\right)$, i.e., $C=C_{t}-C_{0}$. $C_{0}$ ranged from $0.7 \mu \mathrm{l}^{-1}$ to $0.9 \mu \mathrm{l}^{-1}$, representing a percentage between $9 \%$ and $2.5 \%$ of the sediment concentrations measured in the experiments. Each experimental run started at $2.8 \mathrm{~Hz}$, the lowest oscillation frequency of the grid. A steady state was reached after 30 minutes and then after a further 30 minutes (at $t=60$ minutes) the oscillation frequency was increased to $3.3 \mathrm{~Hz}$. A second steady state was reached at $t=90$ minutes, and after a further 30 minutes (at $t=120$ minutes) the frequency was increased to $3.8 \mathrm{~Hz}$. A third steady state was reached at $t=150$ minutes and this continued for a final 30-minute period. Consecutive steady states were reached for frequencies of 4.3 and $4.8 \mathrm{~Hz}$. The evolution of the resuspended sediment concentration $C_{t}$ with time is shown in Fig. 7 for the experiments carried out with both marsh and synthetic sediments for runs with rigid vegetation of $S P F=2.5 \%$. The dashed line in the plot represents the time evolution of the grid oscillation frequencies. Similarly, Oguz et al [58] found that 15 minutes were required for sediment resuspension to reach a steady state in a wave-dominated environment. For the bare soil case, experiments with the different frequencies were also carried out separately (not in the sequence of the increasing frequencies) and the same sediment concentrations were obtained at the steady state. Therefore, all the experiments thereafter were carried out sequentially.

Seven experiments were conducted to study the effect of the consolidation time (runs 21 and 23-28). All of them were carried out without plants, with synthetic sediment and for all the frequencies (Table 2). Three experiments were conducted to study the effect of the sediment type (runs 1, 11 and 21). All of them were carried out without plants for the two days of consolidation time and for all the frequencies (Table 2). Three experiments were conducted to study the effect plant flexibility, rigid plants (run13), flexible plants (run 17) and semi-rigid plants (run 22) have. All the frequencies were considered for runs 13 and 22 (Table 2) and three for run 17. All of them were carried out for $S P F=2.5 \%, 2$ days of consolidation time and for the synthetic sediment. Ten experiments for marsh sediment (runs 1-10) and ten experiments for synthetic sediment (runs 11-20) were conducted to study the effect canopy density and type have on the sediment resuspension.

\section{Results}




\subsection{Vertical turbulent kinetic energy in the presence of a bottom canopy}

402

For experiments without plants, the $T K E$ decreased with vertical distance from the grid (Fig. 8).

For experiments with rigid, semi-rigid or flexible canopies, two layers were distinguished: a transition layer and a within-canopy layer (Fig. 8). Within the canopy layer, the TKE for both the rigid, semi-rigid and flexible canopy $(S P F=2.5 \%)$ cases were below that for the run without plants. The transition layer extended up to at least $6 \mathrm{~cm}$ above the top of the canopy (Fig. 8). In this layer, the TKE for the cases with plants was lower than that for the without-plants case with a TKE difference that decreased from the top of the canopy (38\% lower than for the without plants case) down to $z=10 \mathrm{~cm}$ ( $8.7 \%$ lower than for the without-plants case).

To compare between the runs, the $T K E$ at $z=22 \mathrm{~cm}$ was chosen to represent the $T K E$ within the canopy. In Fig. 9, the TKE is plotted for both rigid (left panel) and flexible (right panel) plants for all the canopy densities studied, and also for the without-plants case. In all cases, the TKE was found to increase with increasing grid oscillation frequency. In both rigid and flexible canopies, the $T K E$ was below that of the without-plants case $(S P F=0 \%)$. In the rigid canopy the $T K E$ reached a minimum at an intermediate value (of $S P F=5 \%$ ), remaining constant afterwards for $S P F>5 \%$. In contrast, for flexible canopies the $T K E$ decreased gradually with increasing $S P F$. It is important to notice that for $S P F<2.5 \%$, flexible and rigid canopies present similar $T K E$ for the same oscillating frequency. However, for $S P F>2.5 \%$, the $T K E$ for flexible plant is smaller than that for rigid plants.

\subsection{Sediment re-suspension in the presence of a canopy: the effect of plant flexibility}

Within the canopy, the behavior of the suspended sediment concentration at the steady state $\left(C_{s s}\right)$ with $S P F$ was different for rigid and flexible canopies (Figs. 10a and 10b, respectively). $C_{s s}$ for the without-plants experiments was greater than for all the experiments with rigid plants. The greater the oscillating frequency, the higher the $C_{s s}$ was. For rigid canopy models, $C_{s s}$ was nearly constant with $S P F$ for all the frequencies tested. In contrast, $C_{S s}$ decreased markedly with SPF for flexible canopies, attaining smaller $C_{s s}$ for the denser flexible canopies than that of the denser rigid canopies of the same SPF. Similar results were obtained for the synthetic sediments for both rigid and flexible plants (Figs. 10c and 10d, respectively). 
$C_{s s}$ was found to follow an exponential relationship with $T K E$ with different exponents for the different vegetation types (Fig. 11). For the same $T K E$, the highest $C_{s s}$ (and the highest coefficient of the exponential) was found for the flexible vegetation model, while the lowest $C_{s s}$ was found for the rigid vegetation model.

\subsection{Sediment resuspension related to sediment bottom consolidation}

In all the experiments, the longer the consolidating time, the lower the $C_{s s}$ was for all the $T K E$ studied (Fig. 12). Two behaviors were observed based on the evolution of $C_{s s}$ with TKE that depended on the consolidation time. The first for the long consolidation time $(>12 \mathrm{~h})$ and the second for the short consolidation time $(<12 \mathrm{~h})$. For long consolidating times above $12 \mathrm{~h}, C_{s s}$ increased with $T K E$, following an exponential dependence. On the other hand, and considering the uncertainties, for bed consolidation times between 1 and 6 hours, $C_{s s}$ was approximately constant with $T K E$.

\subsection{Sediment re-suspension related to sediment bottom characteristics}

The suspended sediment concentration $C_{s s}$ increased exponentially with the $T K E$ for all the sediments tested (Fig. 13). For TKE $<4 \times 10^{-4} \mathrm{~m}^{2} \mathrm{~s}^{-2}$, no differences were obtained between the $C_{S s}$ obtained for the different sediments. In contrast, for $T K E>4 \times 10^{-4} \mathrm{~m}^{2} \mathrm{~s}^{-2}$, the behavior between $C_{s s}$ and the TKE depended on the nature of the sediment. The greatest $C_{s s}$ corresponded to the marsh sediment and the lowest to the synthetic sediment

\section{Discussion}

The bed sediment within non-vegetated and vegetated model canopies were resuspended due to the turbulence generated by the oscillating grid. The resuspension of particles from the sediment beds was found to depend on the characteristics of the structure of the canopy (both plant density and plant flexibility) and the characteristics of the sediment bed (both consolidation time and sediment composition).

\subsection{The effect sediment cohesiveness had on sediment resuspension}

The three cohesive sediments studied were resuspended, due to the turbulence generated by the oscillating grid, producing a homogeneous vertical suspended sediment concentration for all 
the experiments carried out. This homogeneous vertical distribution of sediment is in accordance with the results found by other authors when the suspended sediment concentration was below $80 \mathrm{mg} \mathrm{L}^{-1}$ [59]. In the present study, the maximum concentration of suspended sediment was $30 \mu \mathrm{L} \mathrm{L}{ }^{-1}$, which corresponds to a mass sediment concentration of $75 \mathrm{mg} \mathrm{L}^{-1}$.

The total suspended solids was found to depend on the degree of TKE near the bottom of the bed, as was also found by Tsai and Lick [36]. The turbulent energy dissipation produced by the oscillating grid for the oscillating frequencies studied ranged from $1.02 \times 10^{-4} \mathrm{~m}^{2} \mathrm{~s}^{-3}$ to $5.13 \times 10^{-}$ ${ }^{4} \mathrm{~m}^{2} \mathrm{~s}^{-3}$. This range of turbulence is characteristic of mean turbulence intensities in the shallow littoral zones in lakes, with mean values of $2.41 \times 10^{-4} \mathrm{~m}^{2} \mathrm{~s}^{-3}$ and $3.97 \times 10^{-5} \mathrm{~m}^{2} \mathrm{~s}^{-3}$ for water depths of $0.5 \mathrm{~m}$ and $1.5 \mathrm{~m}$, respectively $[60,61]$. The particle volume concentration was found to exponentially increase with TKE (Fig. 14). The greatest resuspension was found for the marsh sediment, which was $22 \%$ higher than that of the synthetic sediment. Given that the sediment mass was the same for both sediments, it is likely that the higher resuspension rates are associated to the greater concentrations of fine particles in the bed. Then, turbulent events acting on muddy bed substrates produce bed erosion resulting in higher water turbidities than sandier regions under the same hydrodynamic forcing [62]. Therefore, our data show that the greater the concentration of fine particles is in the bottom of the bed, the greater the resuspension of particles in the water column. The increase of fine particles in the water column might cause an increase in water turbidity (i.e. a reduction in water clarity) that may have a negative feedback for the ecosystem, especially for organisms that require light to survive.

\subsection{The effect the structural characteristics of the model canopy had on the resuspension of sediments}

Sediment resuspension depended on the characteristics of the vegetation, which is in accordance with Tinoco and Coco [18]. In the $S P F$ range studied, rigid canopies produced less sediment resuspension than bare soils. This result can be attributed to the reduction of the turbulent kinetic energy by the canopy. However, flexible canopies produce a wide range of resuspended sediment concentrations, expanding from smaller to greater concentrations than those obtained for the rigid canopy and the without-plants case. This behavior can be explained by the movement of the flexible plants' leaves in the water column, because as the leaves are able to capture sediment particles these can be washed off as the flexible plants move. This can 
explain why, for the same $T K E$, flexible plant models produce greater resuspension than rigid models that do not move with the flow. The lower values of the suspended sediment concentration obtained by the flexible canopies compared to the rigid ones, corresponds to the cases with high $S P F$, where the $T K E$ is greater for rigid plants than for flexible plants. Therefore, once sediment particles are resuspended from the bottom their settling in a flexible canopy is lower than it would be in a rigid canopy. Therefore, beds covered with flexible plants in the field might present a greater erosion of the finer particles once resuspended, as they are potentially transported to other regions by waves and currents. In such cases, unlike the beds in rigid canopies, the beds with flexible canopies would result in sandier compositions.

The finding that dense canopies of flexible plants reduces sediment resuspension more than the sparse canopies of flexible plants do, is in accordance with the findings from field $[12,62]$ and laboratory experiments [63]. The presence of macrophytes in shallow lakes effectively abates sediment resuspension as a result of a reduction in bed shear stress or turbulent kinetic energy above the bed $[64,65]$. In experiments conducted in lake enclosures, Li et al [66] found that macrophytes reach their maximum effectiveness in reducing resuspension at a certain speciesspecific biomass threshold, beyond which the biomass effects on resuspension are negligible. This result is in accordance with the findings in the present study. For example, flexible canopies with SPF lower than $\mathrm{SPF}=7.5 \%$ substantially reduce sediment resuspension, whereas canopies with densities over $\mathrm{SPF}=7.5 \%$ do not produce any further decrease in sediment resuspension. In the coastal Mediterranean, canopies of Posidonia oceanica have been found to reduce resuspension rates by three- (medium dense canopies) to seven-fold (dense canopies) compared to those in the adjacent unvegetated floor $[11,12]$.

\subsection{The effect sediment bottom bed consolidation had on sediment resuspension}

Different sediment resuspension dynamics have been found depending on whether the sediment is consolidated for a short or long period. Sediments that have a long consolidation time will require a greater critical turbulent kinetic energy to initiate resuspension from a bed. These results are in accordance with Orlins and Gulliver [35] who found that for $T K E<10^{-3} \mathrm{~m}^{2}$ $\mathrm{s}^{-2}$, the same level of $T K E$ produced a greater resuspension for low consolidation times. Orlins and Gulliver [35] found that for $T K E=10^{-3} \mathrm{~m}^{2} \mathrm{~s}^{-2}$, resuspension did not depend on the consolidation times studied ( 2 and 11 days). Mud erodibility was tested by Lo et al [67] on cores containing suspensions of coastal lake sediments that were consolidated for 1,2 and 4 
weeks, and found that the strengthening of the beds could be attributed to the bed's time consolidation, and inversely on initial suspension concentration over concentrations ranging from fluid mud to hydraulic dredge effluent.

For high TKE of $2 \times 10^{-3} \mathrm{~m}^{2} \mathrm{~s}^{-2}$, Orlins and Gulliver [35] found that the total suspended solids concentration was independent of the consolidation times of the 2 and 11 days they studied. Our experiments were extended to shorter consolidation times than those studied by Orlins and Gulliver [35] but the highest TKE studied was $5.5 \times 10^{-4} \mathrm{~m}^{2} \mathrm{~s}^{-2}$, lower than the threshold found by Orlins and Gulliver [35]. Our results show that the shorter the consolidation time is, the greater the suspended sediment concentration (Fig 11). Furthermore, for consolidation times below $6 \mathrm{~h}$, and considering the uncertainty in the data, the concentration of suspended solids was independent of the TKE for the range of TKE studied. However, for consolidation times above $6 \mathrm{~h}$, the concentration of suspended solids increased with the TKE, especially for $T K E>4 \times 10^{-4} \mathrm{~m}^{2} \mathrm{~s}^{-2}$. For these ranges of consolidation times above $6 \mathrm{~h}$, the difference in the suspended sediment concentration between the different consolidation times decreases with $T K E$ but, contrary to the findings by Orlins and Gulliver [35], still remained different for the highest $T K E$ studied, which was probably due to the fact that the TKE in the present study was below the threshold of Orlins and Gulliver [35]. The results found in our study, agree with those of James et al [68] where, for sediments located at canopy-forming and meadow-forming beds, the concentration of suspended solids increased markedly as a function of increasing bottom shear stress.

\section{Conclusions}

The resuspension of sediment by zero-mean turbulence depends on the consolidation time of the bed, the composition of the sediment and the characteristics of the bed (vegetated or bare soil). For vegetated beds, the characteristics of the canopy, in terms of its plant flexibility, is crucial in determining sediment resuspension. We found that the degree to which the sediment bed was consolidated played a crucial role in determining the magnitude of the sediment resuspension. Sediments that have a long consolidation time will require a greater critical turbulent kinetic energy to initiate resuspension from a bed. As such, for beds with consolidation times lower than six hours, the suspended solids were independent of the turbulent kinetic energy. However, for consolidation times above six hours, the concentration of the resuspended sediment increased markedly with the turbulent kinetic energy, especially 
567 for turbulent kinetic energies greater than $4 \times 10^{-4} \mathrm{~m}^{2} \mathrm{~s}^{-2}$. For these ranges of consolidation times, 568 the suspended sediment concentrations increased with the turbulent kinetic energies.

570 In the simulated vegetated experiments, rigid, semi-rigid and flexible plant canopies were 571 found to reduce the turbulent kinetic energy in shear-free conditions compared to without572 plants cases. Dense flexible canopies of $\mathrm{SPF}=5 \%$ reduced the turbulent kinetic energy more 573 than the rigid canopies, thus reducing sediment resuspension in the water column. In contrast, 574 sparse canopies of flexible stems produced similar turbulent kinetic energies to those of the 575 rigid canopies of the same density For the same level of turbulent kinetic energy the 576 resuspended sediment in the flexible canopies was higher than in the rigid canopies as a result 577 of the movement of the plant leaves. Assuming that stable substrates play a vital role for plant 578 survival, this suggests a mechanism that may lead to dense distributions of flexible vegetation 579 being better able to survive than sparse flexible canopies.

580

581 
583

584

585

586

587

588

589

590

591

592

593

594

595

596

597

598

599

600

601

602

603

604

605

606

607

608

609

610

611

612

613

614

615

616

617

1. Vermaat J, Santamaria L, Roos P (2000) Water flow across and sediment trapping in submerged macrophyte beds of contrasting growth form. Arch fur Hydrobiol 148:549562

2. Madsen JD, Chambers P a, James WF, et al (2001) The interaction between water movement, sediment dynamics and submersed macrophytes. Hydrobiologia 444:7184

3. Pujol D, Colomer J, Serra T, Casamitjana X (2010) Effect of submerged aquatic vegetation on turbulence induced by an oscillating grid. Cont Shelf Res 30:1019-1029

4. Ward L, Kemp W, Boynton W (1984) The influence of waves and seagrass communities on suspended particulates in an estuarine embayment. Mar Geol 59:85103

5. Koch EW (2001) Beyond Light: Physical, Geological, and Geochemical Parameters as Possible Submersed Aquatic Vegetation Habitat Requirements. Estuaries 24:1 . doi: $10.2307 / 1352808$

6. de Boer WF (2007) Seagrass-sediment interactions, positive feeedbacks and cretical thresholds for occurrence: a review. Hydrobiologia 591:5-24

7. Carr J, D’Odorico P, McGlathery K, Wiberg P (2010) Stability and bistability of seagrass ecosystems in shallow coastal lagoons: Role of feedbacks with sediment resuspension and light attenuation. J Geophys Res Biogeosciences 115:1-14 . doi: 10.1029/2009JG001103

8. Van Der Heide T, Van Nes EH, Geerling GW, et al (2007) Positive feedbacks in seagrass ecosystems: Implications for success in conservation and restoration. Ecosystems 10:1311-1322 . doi: 10.1007/s10021-007-9099-7

9. Zhu M, Zhu G, Nurminen L, et al (2015) The influence of macrophytes on sediment resuspension and the effect of associated nutrients in a shallow and Large Lake (Lake Taihu, China). PLoS One 10:1-20 . doi: 10.1371/journal.pone.0127915

10. Wu T, Timo H, Qin B, et al (2016) In-situ erosion of cohesive sediment in a large shallow lake experiencing long-term decline in wind speed. J Hydrol 539:254-264 . doi: 10.1016/j.jhydrol.2016.05.021

11. Gacia E, Duarte CM (2001) Sediment retention by a Mediterranean Posidonia oceanica meadow: The balance between deposition and resuspension. Estuar Coast Shelf Sci 52:505-514

12. Granata TC, Serra T, Colomer J, et al (2001) Flow and particle distributions in a nearshore seagrass meadow before and after a storm. Mar Ecol Prog Ser 218:95-106

13. Pujol D, Serra T, Colomer J, Casamitjana X (2013) Flow structure in canopy models 
14. Pujol D, Casamitjana X, Serra T, Colomer J (2013) Canopy-scale turbulence under oscillatory flow. Cont Shelf Res 66:9-18 . doi: http://dx.doi.org/10.1016/j.csr.2013.06.012

15. Ros À, Colomer J, Serra T, et al (2014) Experimental observations on sediment resuspension within submerged model canopies under oscillatory flow. Cont Shelf Res 91:220-231

16. Ondiviela B, Losada IJ, Lara JL, et al (2014) The role of seagrasses in coastal protection in a changing climate. Coast Eng 87:158-168 . doi: 10.1016/j.coastaleng.2013.11.005

17. Black KS, Tolhurst TJ, Paterson DM, Hagerthey SE (2002) Working with Natural Cohesive Sediments. J Hydraul Eng 128:2-8 . doi: 10.1061/(ASCE)07339429(2002)128:1(2)

18. Tinoco RO, Coco G (2016) A laboratory study on sediment resuspension within arrays of rigid cylinders. Adv Water Resour 92:1-9 . doi: 10.1016/j.advwatres.2016.04.003

19. Yang Y, Wang YP, Gao S, et al (2016) Sediment resuspension in tidally dominated coastal environments: new insights into the threshold for initial movement. Ocean Dyn 66:401-417 . doi: 10.1007/s10236-016-0930-6

20. Horppila J, Kaitaranta J, Joensuu L, Nurminen L (2013) Influence of emergent macrophyte (Phragmites australis) density on water turbulence and erosion of organicrich sediment. J Hydrodyn Ser B 25:288-293 . doi: 10.1016/S1001-6058(13)60365-0

21. Bouma T, Friedrichs M, Klaassen P, et al (2009) Effects of shoot stiffness, shoot size and current velocity on scouring sediment from around seedlings and propagules. Mar Ecol Prog Ser 388:293-297 . doi: 10.3354/meps08130

22. Lawson S, Wiberg P, McGlathery K, Fugate D (2007) Wind-driven sediment suspension controls light availability in a shallow coastal lagoon. Estuaries and Coasts 30:102 . doi: 10.1007/bf02782971

23. Hansen JCR, Reidenbach M a. (2013) Seasonal Growth and Senescence of a Zostera marina Seagrass Meadow Alters Wave-Dominated Flow and Sediment Suspension Within a Coastal Bay. Estuaries and Coasts 36:1099-1114 . doi: 10.1007/s12237-0139620-5

24. Mendez F, Losada I, Losada M (1999) Hydrodynamics induced by wind waves in a vegetation field. J Geophys Res - Ocean 104:18383-18396

25. Nepf HM (1999) Drag, turbulence, and diffusion in flow through emergent vegetation. Water Resour Res

26. Nepf HM, Vivoni E (2000) Flow structure in depth-limited, vegetated flow. J Geophys Res 105:28547-28557 
27. Poggi D, Porporato A, Ridolfi L, et al (2003) The effect of vegetation density on canopy sub-layer turbulence. Boundary-Layer Meteorlogy 111:565-587

28. Neumeier U (2007) Velocity and turbulence variations at the edge of saltmarshes. Cont Shelf Res 27:1046-1059 . doi: 10.1016/j.csr.2005.07.009

29. Coates MJ, Folkard AM (2009) The effects of littoral zone vegetation on turbulent mixing in lakes. Ecol Modell 220:2726

30. De Silva IP., Fernando HJS (1994) Oscillating grids as a source of nearly isotropic turbulence. Phys Fluids 6:2455-2464

31. Colomer J, Peters F, Marrasé C (2005) Experimental analysis of coagulation of particles under low-shear flow. Water Res 39:2994-3000 . doi: 10.1016/j.watres.2005.04.076

32. Serra T, Colomer J, Logan BE (2008) Efficiency of different shear devices on flocculation. Water Res 42:1113-1121

33. Nokes R (1988) On the entrainmnent rate across a density interface. J Fluid Mech 188:185-204

34. Holzner M, Liberzon A, Guala M, et al (2006) Generalized detection of a turbulent front generated by an oscillating grid. Exp Fluids 41:711-719 . doi: 10.1007/s00348006-0193-y

35. Orlins JJ, Gulliver JS (2003) Turbulence quantification and sediment resuspension in an oscillating grid chamber. Exp Fluids 34:662-677 . doi: 10.1007/s00348-003-0595-z

36. Tsai C-H, Lick W (1986) A Portable Device for Measuring Sediment Resuspension. J Great Lakes Res 12:314-321 . doi: 10.1016/S0380-1330(86)71731-0

37. Huppert HE, Turner JS, Hallworth MA (1995) Sedimentation and entrainment in dense layers of suspended particles stirred by an oscillating grid. J Fluid Mech 289:263

38. El Allaoui N, Serra T, Soler M, et al (2015) Modified hydrodynamics in canopies with longitudinal gaps exposed to oscillatory flows. J Hydrol 531:840-849

39. Redondo JM, De Madron XD, Medina P, et al (2001) Comparison of sediment resuspension measurements in sheared and zero-mean turbulent flows. Cont Shelf Res 21:2095-2103 . doi: 10.1016/S0278-4343(01)00044-9

40. Ghisalberti M, Nepf HM (2002) Mixing layers and coherent structures in vegetated aquatic flows. 107:

41. Folkard AM (2005) Hydrodynamics of model Posidonia oceanica patches in shallow water. Limnol Oceanogr 50:1592-1600

42. El Allaoui N, Serra T, Colomer J, et al (2016) Interactions between fragmented seagrass canopies and the local hydrodynamics. PLoS One 11:1-19 . doi: 
43. Guillén JE, Sánchez JL, Jiménez S, et al (2013) Evolution of Posidonia oceanica seagrass meadows and its implications for management. J Sea Res 83:65-71 . doi: 10.1016/j.seares.2013.04.012

44. Rupprecht F, Möller I, Paul M, et al (2017) Vegetation-wave interactions in salt marshes under storm surge conditions. Ecol Eng 100:301-315 . doi: 10.1016/j.ecoleng.2016.12.030

45. Pedlow CL, Dibble ED, Getsinger KD (2006) Littoral habitat heterogeneity and shifts in plant composition relative to a fall whole-lake fluridone application in Perch lake, Michigan. J Aquat Plant Manag 44:26-31

46. Serra T, Fernando HJS, Rodríguez R V (2004) Effects of emergent vegetation on lateral diffusion in wetlands. Water Res 38:139-47

47. Neumeier U, Amos CL (2006) Turbulence reduction by the canopy of coastal Spartina salt-marshes. J Coast Res 39:433-439

48. Serra T, Granata T, Colomer J, et al (2003) The role of advection and turbulent mixing in the vertical distribution of phytoplankton. Estuar Coast Shelf Sci 56:53-62 . doi: $10.1016 / \mathrm{S} 0272-7714(02) 00120-8$

49. Serra T, Soler M, Julia R, et al (2005) Behaviour and dynamics of a hydrothermal plume in Lake Banyoles, Catalonia, NE Spain. Sedimentology 52:795-808

50. Rijn LC Van (2007) Unified View of Sediment Transport by Currents and Waves . I : Initiation of Motion, Bed Roughness , and Bed-Load Transport. J Hydraul Eng 133:649-667

51. Blott SJ, Pye K (2012) Particle size scales and classification of sediment types based on particle size distributions: Review and recommended procedures. Sedimentology 59:2071-2096 . doi: 10.1111/j.1365-3091.2012.01335.x

52. Goring DG, Nikora VI (2002) Despiking acoustic doppler velocimeter data. J Hydraul Eng 128:117-126

53. Hopfinger E, Toly J (1976) Spatially decaying turbulence and its relation to mixing across density interfaces. J Fluid Mech 78:155-175

54. Matsunaga N, Sugihara Y, Komatsu T, Masuda A (1999) Quantitative properties of oscillating-grid turbulence in a homogeneous fluid. Fluid Dyn Res 25:147-165

55. Wan Mohtar WHM (2016) Oscillating-grid turbulence at large strokes: Revising the equation of Hopfinger and Toly. J Hydrodyn 28:473-481

56. Rotach MW (1993) Turbulence close to a rough urban surface. Part I: Reynolds stress. Boundary-Layer Meteorlogy 65:1-28 
58. Oguz E, Elginoz N, Koroglu A, Kabdasli MS (2013) The effect of reed beds on wave attenuation and suspended sediment concentration. J Coast Res 65:356-361 . doi: 10.2112/SI65-061.1

59. Green MO, Coco G (2013) Review of wave-driven sediment resuspension and transport in estuaries. Rev Geophys 52:77-117

60. G.-Tóth L, Parpala L, Balogh C, et al (2011) Zooplankton community response to enhanced turbulence generated by water-level decrease in Lake Balaton, the largest shallow lake in Central Europe. Limnol Oceanogr 56:2211-2222 . doi: 10.4319/lo.2011.56.6.2211

61. Zhou J, Qin B, Han X (2017) The synergetic effects of turbulence and turbidity on the zooplankton community structure in large, shallow Lake Taihu. Environ Sci Pollut Res 25:1168-1175 . doi: 10.1007/s11356-017-0262-1

62. Zikhali V, Tirok K, Stretch D (2015) Sediment resuspension in a shallow lake with muddy substrates: St Lucia, South Africa. Cont Shelf Res 108:112-120 . doi: 10.1016/j.csr.2015.08.012

63. Wu D, Hua Z (2014) The effect of vegetation on sediment resuspension and phosphorus release under hydrodynamic disturbance in shallow lakes. Ecol Eng 69:55-62 . doi: 10.1016/j.ecoleng.2014.03.059

64. Hendriks IE, Sintes T, Bouma TJ, Duarte CM (2008) Experimental assessment and modeling evaluation of the effects of the seagrass Posidonia oceanica on flow and particle trapping. Mar Ecol Prog Ser 356:163-173

65. Chen T, Xu Y, Zhu S, Cui F (2015) Combining physico-chemical analysis with a Daphnia magna bioassay to evaluate a recycling technology for drinking water treatment plant waste residuals. Ecotoxicol Environ Saf 122:368-376 . doi: 10.1016/j.ecoenv.2015.08.023

66. Li EH, Li W, Liu GH, Yuan LY (2008) The effect of different submerged macrophyte species and biomass on sediment resuspension in a shallow freshwater lake. Aquat Bot 88:121-126 . doi: 10.1016/j.aquabot.2007.09.001

67. Lo EL, Bentley SJ, Xu K (2014) Experimental study of cohesive sediment consolidation and resuspension identifies approaches for coastal restoration: Lake Lery, Louisiana. Geo-Marine Lett 34:499-509 . doi: 10.1007/s00367-014-0381-3

68. James CS, Birkhead AL, Jordanova AA, O'Sullivan JJ (2004) Flow resistance of emergent vegetation. J Hydraul Eng 42:390-398 
Table 1. Characteristics of the sediment types used in the experimental work

SEDIMENT NAME

MARSH

SYNTHETIC

LAKE

\section{ORIGIN}

Ter Natural Park (NE Catalonia, Spain)

ISO12103-1, A4 coarse. Powder

Technology Inc. Burnsville

Lake Banyoles (NE Catalonia, Spain)

762

763

Table 2. Summary of experimental conditions and parameters. SPF represents the solid plant fraction (see Section 2.2), $n$ is the canopy density (shoots per square meter), vegetation type, consolidation time, sediment type and oscillating grid frequency $(f)$.

\begin{tabular}{|c|c|c|c|c|c|c|}
\hline Run & $\begin{array}{l}\text { SPF } \\
(\%)\end{array}$ & $\begin{array}{l}\text { n } \\
\left(\text { shoots } \mathbf{m}^{-2}\right)\end{array}$ & $\begin{array}{l}\text { Vegetation } \\
\text { type }\end{array}$ & $\begin{array}{l}\text { Consolidation } \\
\text { time (days) }\end{array}$ & Sediment type & $f(\mathbf{H z})$ \\
\hline 1 & 0 & 0 & - & 2 & Marsh & $2.8,3.3,3.8,4.3,4.8$ \\
\hline 2 & 1 & 354 & Rigid & 2 & Marsh & $2.8,3.8,4.8$ \\
\hline 3 & 2.5 & 884 & Rigid & 2 & Marsh & $2.8,3.3,3.8,4.3,4.8$ \\
\hline 4 & 5 & 1768 & Rigid & 2 & Marsh & $2.8,3.8,4.8$ \\
\hline 5 & 7.5 & 2652 & Rigid & 2 & Marsh & $2.8,3.8,4.8$ \\
\hline 6 & 10 & 3537 & Rigid & 2 & Marsh & $2.8,3.8,4.8$ \\
\hline 7 & 2.5 & 884 & Flexible & 2 & Marsh & $2.8,3.8,4.8$ \\
\hline 8 & 5 & 1768 & Flexible & 2 & Marsh & $2.8,3.8,4.8$ \\
\hline 9 & 7.5 & 2652 & Flexible & 2 & Marsh & $2.8,3.8,4.8$ \\
\hline 10 & 10 & 3537 & Flexible & 2 & Marsh & $2.8,3.8,4.8$ \\
\hline 11 & 0 & 0 & - & 2 & Synthetic & $2.8,3.3,3.8,4.3,4.8$ \\
\hline 12 & 1 & 354 & Rigid & 2 & Synthetic & $2.8,3.8,4.8$ \\
\hline 13 & 2.5 & 884 & Rigid & 2 & Synthetic & $2.8,3.3,3.8,4.3,4.8$ \\
\hline 14 & 5 & 1768 & Rigid & 2 & Synthetic & $2.8,3.8,4.8$ \\
\hline 15 & 7.5 & 2652 & Rigid & 2 & Synthetic & $2.8,3.8,4.8$ \\
\hline 16 & 10 & 3537 & Rigid & 2 & Synthetic & $2.8,3.8,4.8$ \\
\hline 17 & 2.5 & 884 & Flexible & 2 & Synthetic & $2.8,3.8,4.8$ \\
\hline 18 & 5 & 1768 & Flexible & 2 & Synthetic & $2.8,3.8,4.8$ \\
\hline 19 & 7.5 & 2652 & Flexible & 2 & Synthetic & $2.8,3.8,4.8$ \\
\hline
\end{tabular}




\begin{tabular}{lllllll}
\hline $\mathbf{2 0}$ & 10 & 3537 & Flexible & 2 & Synthetic & $2.8,3.8,4.8$ \\
\hline $\mathbf{2 1}$ & 0 & 0 & - & 2 & Lake & $2.8,3.3,3.8,4.3,4.8$ \\
\hline $\mathbf{2 2}$ & 2.5 & 884 & $\begin{array}{l}\text { Semi- } \\
\text { rigid }\end{array}$ & 2 & Synthetic & $2.8,3.3,3.8,4.3,4.8$ \\
& & & - & 0.042 & Synthetic & $2.8,3.3,3.8,4.3,4.8$ \\
\hline $\mathbf{2 3}$ & 0 & 0 & - & 0.125 & Synthetic & $2.8,3.3,3.8,4.3,4.8$ \\
\hline $\mathbf{2 4}$ & 0 & 0 & - & 0.25 & Synthetic & $2.8,3.3,3.8,4.3,4.8$ \\
\hline $\mathbf{2 5}$ & 0 & 0 & - & 0.5 & Synthetic & $2.8,3.3,3.8,4.3,4.8$ \\
\hline $\mathbf{2 6}$ & 0 & 0 & - & 1 & Synthetic & $2.8,3.3,3.8,4.3,4.8$ \\
\hline $\mathbf{2 7}$ & 0 & 0 & - & 3 & Synthetic & $2.8,3.3,3.8,4.3,4.8$ \\
\hline $\mathbf{2 8}$ & 0 & 0 & - & & & \\
\hline
\end{tabular}




\section{Captions to figures}

769

770

771

772

773

774

775

776

777

778

779

780

781

782

783

784

785

786

787

788

789

790

791

792

793

794

795

796

797

798

Figure 1. Schematic diagram of the experimental OGT setup (top panel). Photograph of the grid (bottom panel).

Figure 2. Vegetation simulations: (a) rigid vegetation; (b) flexible vegetation and (c) semi-rigid vegetation, and the plant distribution for the range of canopy densities studied: (d) $S P F=1 \%$, (e) $S P F=2.5 \%$, (f) $S P F=5 \%$, (g) $S P F=7.5 \%$ and (h) $S P F=10 \%$.

Figure 3. Lateral obstruction area of the vegetation calculated from lateral pictures of a 2.5

cm thick canopy for (a) flexible plants and (b) rigid plants, for different SPF.

Figure 4. Particle size distribution of the synthetic, lake and salt marsh sediments used in the experiments. The vertical dashed lines represent the classification by Rijn (2007).

Figure 5. Particle sediment concentration within the suspension versus Turbulent Kinetic Energy for the three bed loads of 100, 200, and $300 \mathrm{gL}^{-1}$ (Experiment with no vegetation and a time consolidation bed of two days for synthetic sediment).

Figure 6. Relationship between the total kinetic energy $(K E)$ at $\mathrm{z}=22 \mathrm{~cm}$ and the solid plant fraction $(S P F)$ of the canopies for oscillating frequencies, $\mathrm{f}=2.8,3.8$ and $4.8 \mathrm{~Hz}$, for (a) rigid and (b) flexible canopies. Horizontal dashed line corresponds to the $A D V$ noise level for the $K E$, set at $0.44 \mathrm{~cm}^{2} \mathrm{~s}^{-1}$. c) TKE versus $\left(\mathrm{z} / \mathrm{h}_{\mathrm{s}}\right)^{-2}$ for the case WP and for $\mathrm{RV}$ and $\mathrm{FV}$ of $\mathrm{SPF}=5 \%$. Lines represent the linear fit between TKE and $\left(\mathrm{z} / \mathrm{h}_{\mathrm{s}}\right)^{-2}$. For the WP case TKE $=7.82\left(\mathrm{z} / \mathrm{h}_{\mathrm{s}}\right)^{-2}$ $11.08\left(\mathrm{R}^{2}=0.9987\right)$, for the RV case TKE=6.76( $\left.\mathrm{z} / \mathrm{h}_{\mathrm{s}}\right)^{-2}-5.17\left(\mathrm{R}^{2}=0.9954\right)$ and for the FV case $\mathrm{TKE}=2.69\left(\mathrm{z} / \mathrm{h}_{\mathrm{s}}\right)^{-2}-2.37\left(\mathrm{R}^{2}=0.9476\right)$.

Figure 7. Time evolution of the sediment concentration for experiments carried out for rigid vegetation with $S P F=2.5 \%$, for the synthetic sediment and the marsh sediment. The dashed line at the top panel corresponds to the evolution of the oscillation frequency (f) over the full time period of each experiment run. 
Figure 8. TKE profiles for experimental runs without plants $(W P)$, and with flexible (FV), rigid $(\mathrm{RV})$ and semi-rigid vegetation (SMRV), all with $S P F=2.5 \%$. Grid oscillation frequency was $f=4.8 \mathrm{~Hz}$ in all cases shown.

Figure 9. Relationship between the turbulent kinetic energy (TKE) at $z=22 \mathrm{~cm}$ and the solid plant fraction $(S P F)$ of the canopies for different oscillating grid frequencies, $f$, for (a) rigid and (b) flexible canopies.

Figure 10. Relationship between the suspended sediment concentration at the steady state $\left(C_{s s}\right)$ measured at $z=0.22 \mathrm{~m}$ and the solid plant fraction $(S P F)$ for different oscillating frequencies (f) for (a and c) rigid, (b and d) flexible canopies, for the marsh (top) and synthetic sediment (bottom).

Figure 11. Dependence of the sediment concentration on the suspension at $z=22 \mathrm{~cm}$ (i.e. $\left.z / h_{s}=0.7\right)$ and the turbulent kinetic energy, for the three types of canopies (rigid, semi-rigid and flexible) for a solid plant fraction of $2.5 \%$. For all runs, a two-day synthetic consolidated bed was used. Vertical error bars are calculated from the standard deviation of different measurements of the same run. Solid lines represent the exponential best fit curve through the data obtained in each case. The equations of the exponential fitting are $C_{s s}=1.46 \mathrm{e}^{7448 T K E}$ $\left(\mathrm{r}^{2}=0.9968\right)$ for FV, $C_{s s}=0.87 \mathrm{e}^{7085 T K E}\left(\mathrm{r}^{2}=0.9932\right)$ for $\mathrm{SMRV}$ and $C_{S S}=1.49 \mathrm{e}^{2733 T K E}\left(\mathrm{r}^{2}=0.9622\right)$ for RV.

Figure 12. Relationship between the sediment concentration of the suspension at $z=22 \mathrm{~cm}$ (i.e. $\left.z / h_{s}=0.7\right)$ and the turbulent kinetic energy, for the seven bed consolidation times, varying from one hour to three days. For all runs, the synthetic type sediment was used. Vertical error bars are calculated from the standard deviation of different measurements of the same run.

Figure 13. Relationship between the sediment concentration $C_{s s}$ at $z=22 \mathrm{~cm}$ at the steady state and the turbulent kinetic energy, for the three types of sediments (synthetic, lake and marsh) for the without-plants experiments. For all runs, a two-day consolidated bed was used. Vertical error bars are calculated from the standard deviation of different measurements of the same run. Solid lines represent the exponential best fit curve through the data obtained in each case. The equations of the exponential fitting are $C_{S s}=0.56 \mathrm{e}^{5937 T K E}\left(\mathrm{r}^{2}=0.9798\right)$ for the marsh 
832 sediment, $C_{S s}=0.67 \mathrm{e}^{5213 T K E}\left(\mathrm{r}^{2}=0.9644\right)$ for the lake sediment and $C_{S s}=0.94 \mathrm{e}^{4139 T K E}\left(\mathrm{r}^{2}=0.9398\right)$

833 for the synthetic sediment.

834

835 Figure 14. Relationship between the sediment concentration of the suspension at $z=22 \mathrm{~cm}$ (i.e. $\left.836 z / h_{s}=0.7\right)$ and the turbulent kinetic energy, for the rigid vegetation runs, no vegetation runs and 837 for flexible vegetation, for both the synthetic and marsh sediment. For all runs, a two-day 838 consolidated bed was used. Solid lines represent the exponential best fit curve through the 839 obtained data in each case. The equations of the exponential fitting are $C_{s s}=0.7 \mathrm{e}^{5444 T K E}$ $840\left(\mathrm{r}^{2}=0.9073\right)$ for $\mathrm{RV}$, and $C_{s s}=1.09 \mathrm{e}^{10012 T K E}\left(\mathrm{r}^{2}=0.8770\right)$ for $\mathrm{FV}$. 


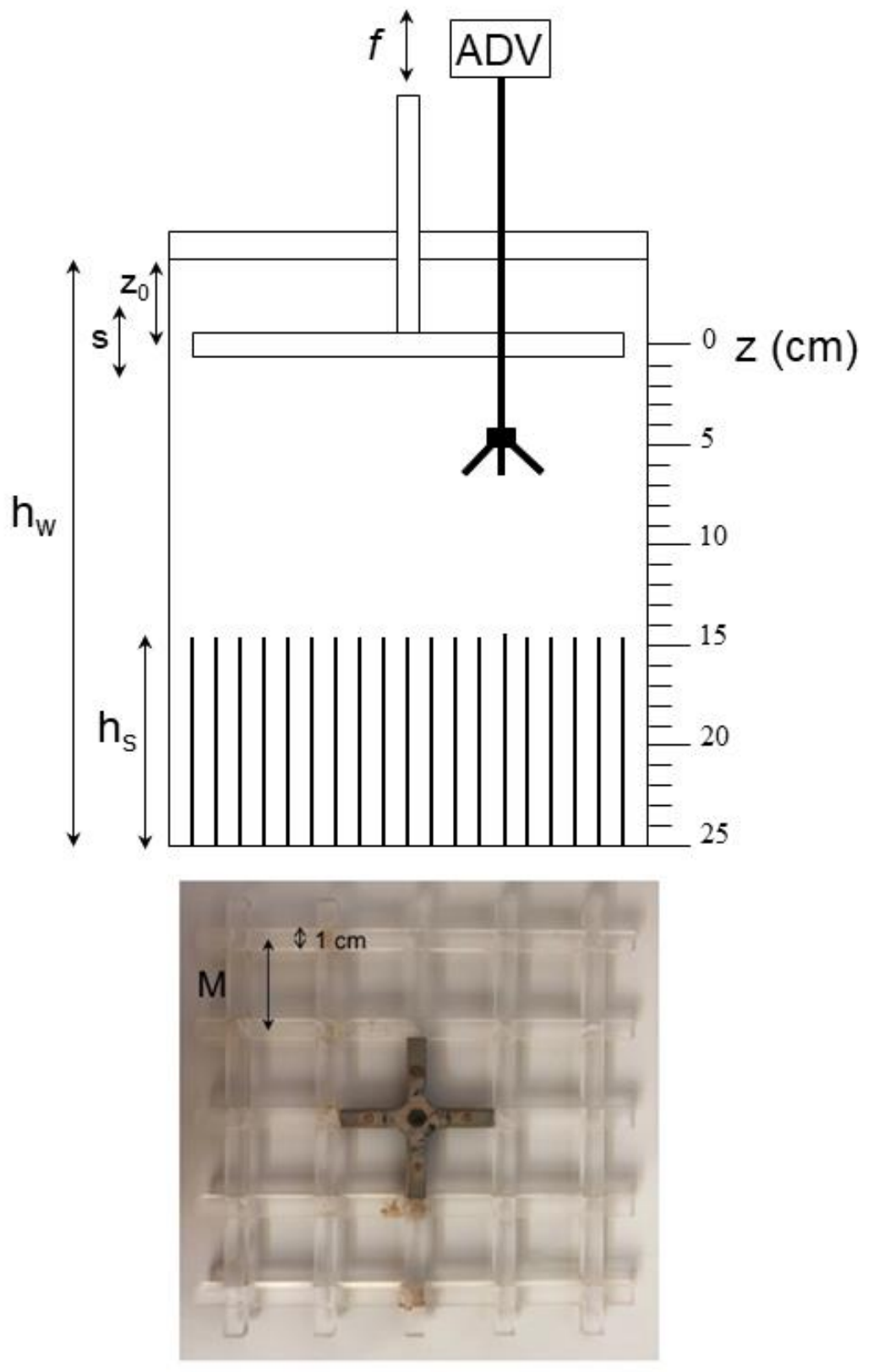

Figure 1. Schematic diagram of the experimental OGT setup (top panel). Photograph of the grid (bottom panel). 


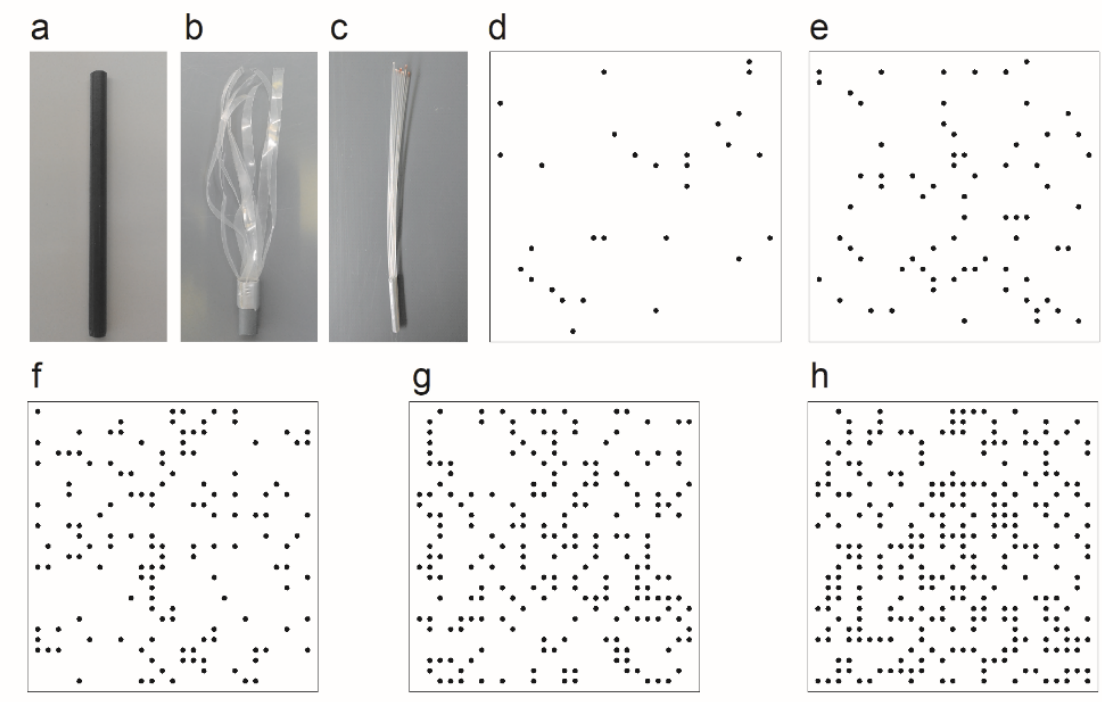

Figure 2. Vegetation simulations: (a) rigid vegetation; (b) flexible vegetation and (c) semi rigid vegetation, and the plant distribution for the range of canopy densities studied: (d) SPF $=1 \%$, (e) $\mathrm{SPF}=2.5 \%$, (f) $\mathrm{SPF}=5 \%$, (g) $\mathrm{SPF}=7.5 \%$ and (h) $\mathrm{SPF}=10 \%$. 

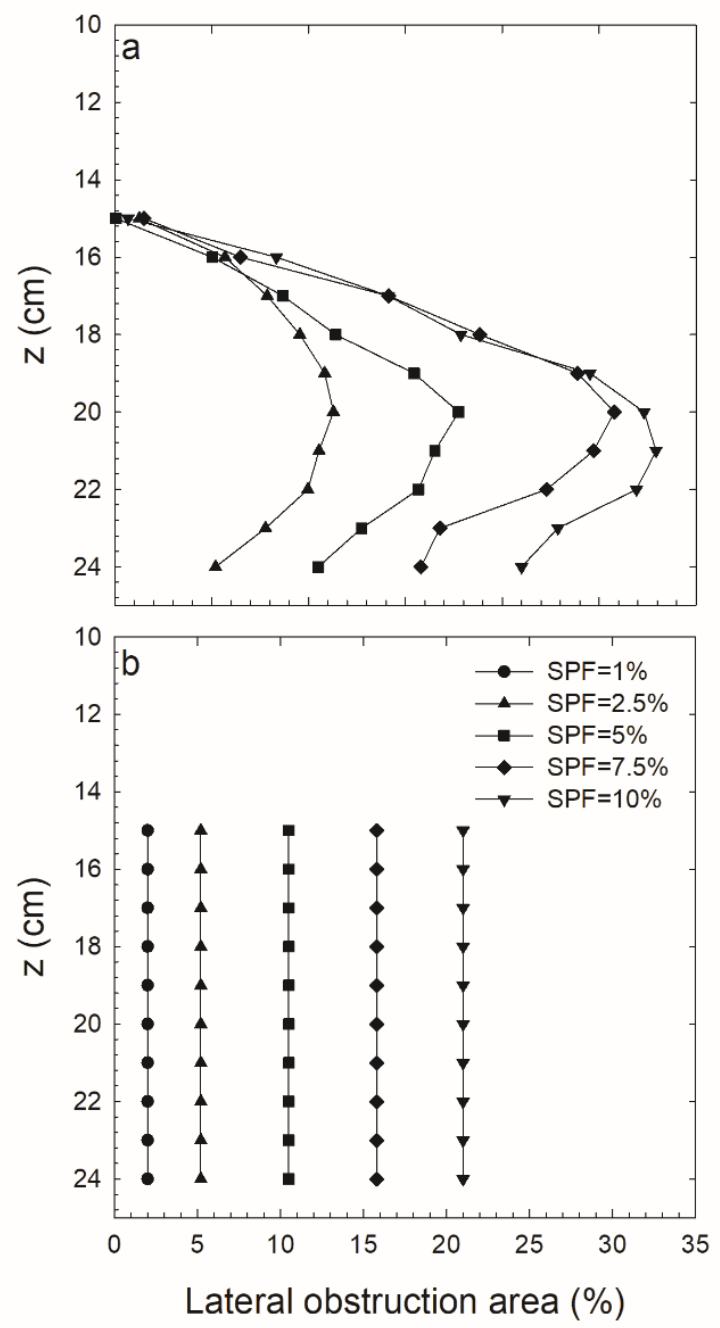

Figure 3. Lateral obstruction area of the vegetation calculated from lateral pictures of a 2.5 $\mathrm{cm}$ thick canopy for (a) flexible plants and (b) rigid plants, for different SPF. 


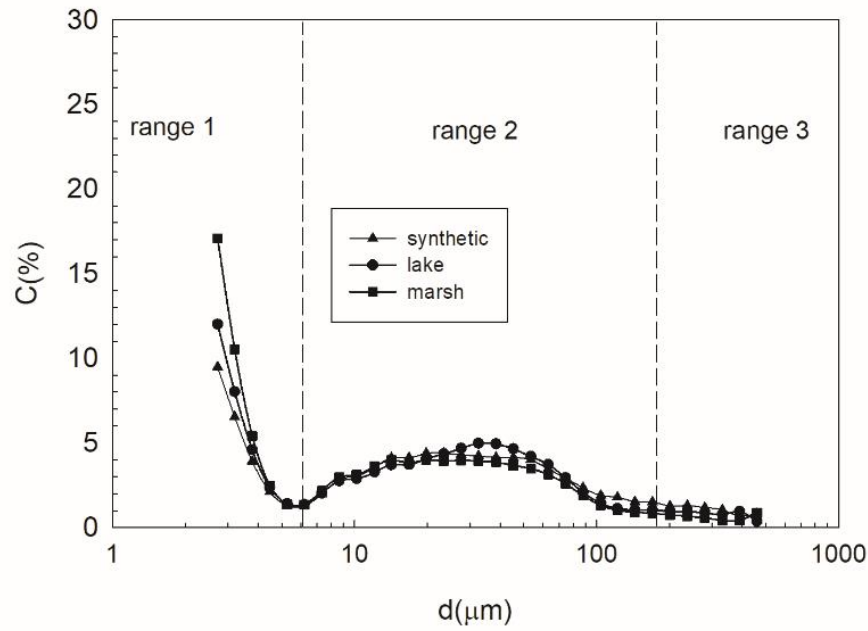

Figure 4. Particle size distribution of the synthetic, lake and salt marsh sediments used in the experiments. The vertical dashed lines represent the classification by Rijn (2007). 


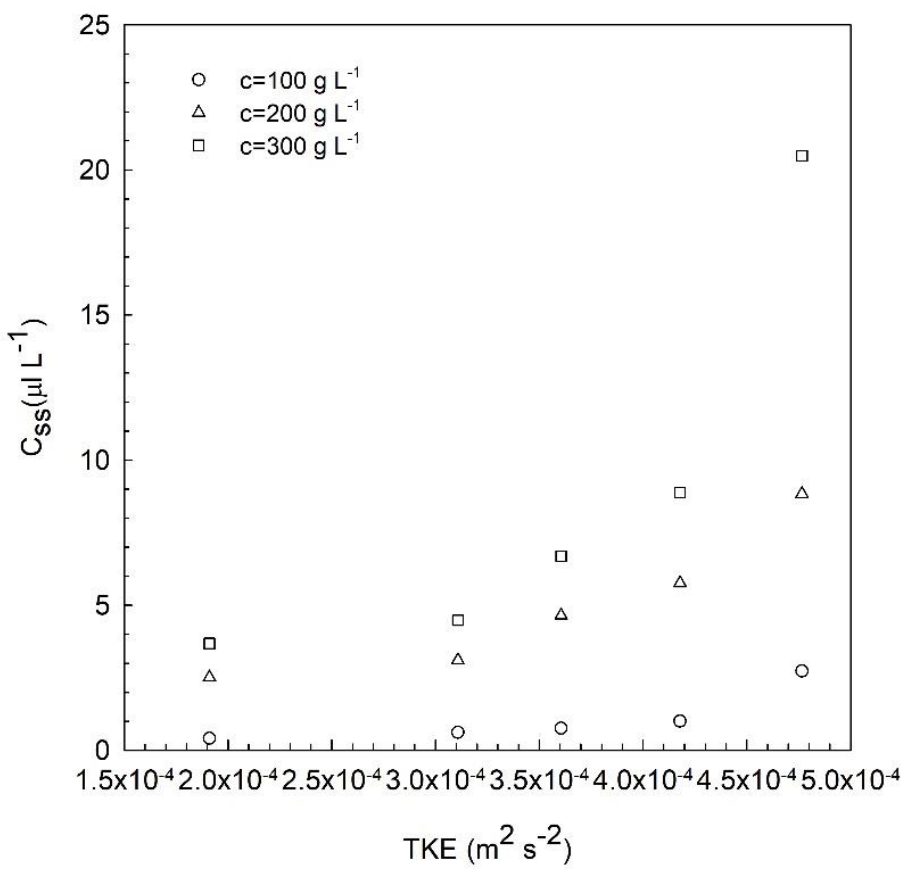

Figure 5. Particle sediment concentration within the suspension versus Turbulent Kinetic Energy for the three bead loads of 100, 200, and $300 \mathrm{gL}^{-1}$ (Experiment with no vegetation and a time consolidation bed of two days for synthetic sediment). 

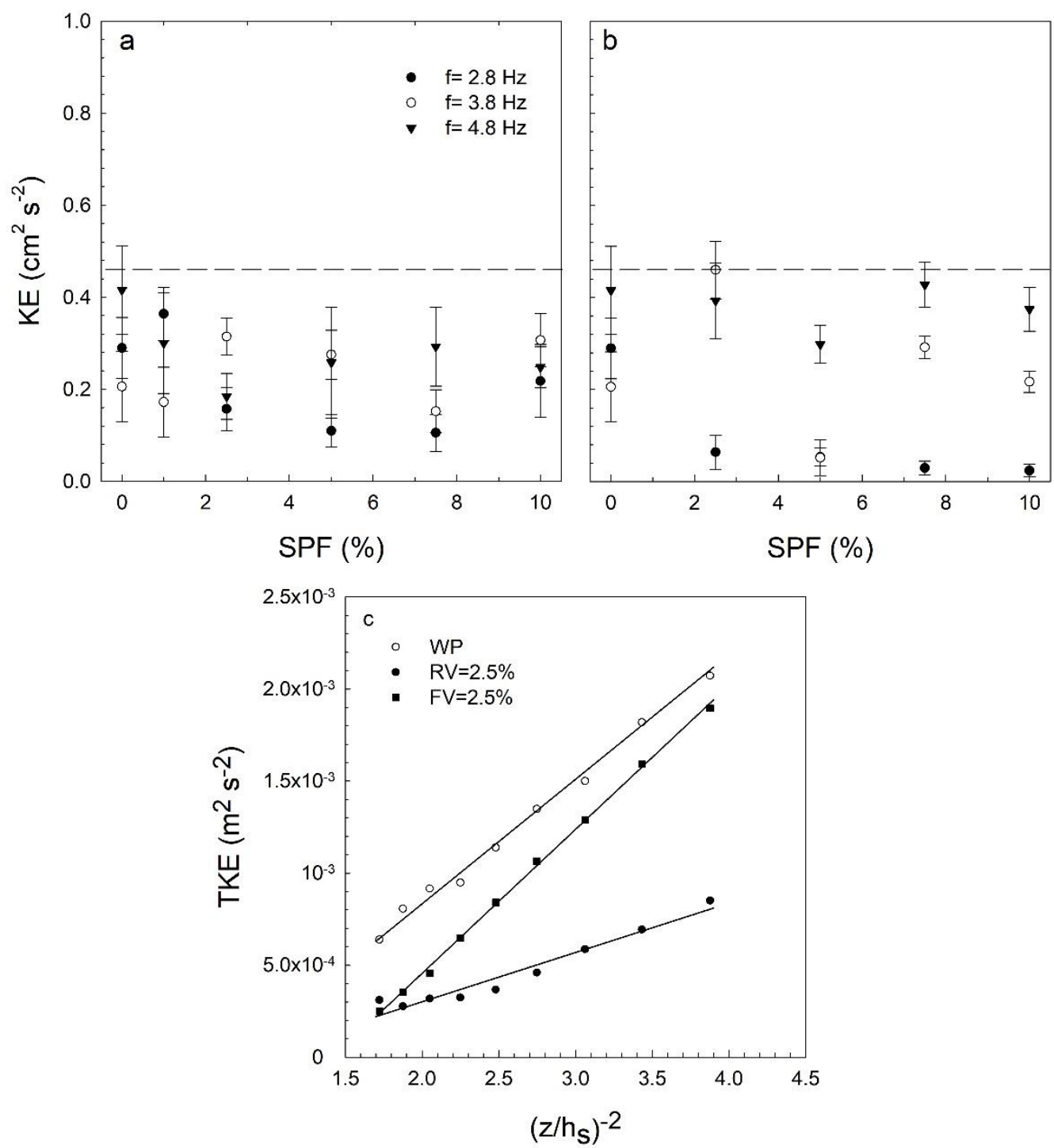

Figure 6. Relationship between the total kinetic energy $(K E)$ at $\mathrm{z}=22 \mathrm{~cm}$ and the solid plant fraction $(S P F)$ of the canopies for oscillating frequencies, $\mathrm{f}=2.8,3.8$ and $4.8 \mathrm{~Hz}$, for (a) rigid and (b) flexible canopies. Horizontal dashed line corresponds to the $A D V$ noise level for the $K E$, set at $0.44 \mathrm{~cm}^{2} \mathrm{~s}^{-1}$. c) TKE versus $\left(\mathrm{z} / \mathrm{h}_{\mathrm{s}}\right)^{-2}$ for the case WP and for $\mathrm{RV}$ and $\mathrm{FV}$ of $\mathrm{SPF}=5 \%$. Lines represent the linear fit between TKE and $\left(\mathrm{z} / \mathrm{h}_{\mathrm{s}}\right)^{-2}$. For the WP case TKE $=7.82\left(\mathrm{z} / \mathrm{h}_{\mathrm{s}}\right)^{-2}-$ $11.08\left(R^{2}=0.9987\right)$, for the RV case TKE $=6.76\left(\mathrm{z} / \mathrm{h}_{\mathrm{s}}\right)^{-2}-5.17\left(\mathrm{R}^{2}=0.9954\right)$ and for the FV case $\mathrm{TKE}=2.69\left(\mathrm{z} / \mathrm{h}_{\mathrm{s}}\right)^{-2}-2.37\left(\mathrm{R}^{2}=0.9476\right)$. 


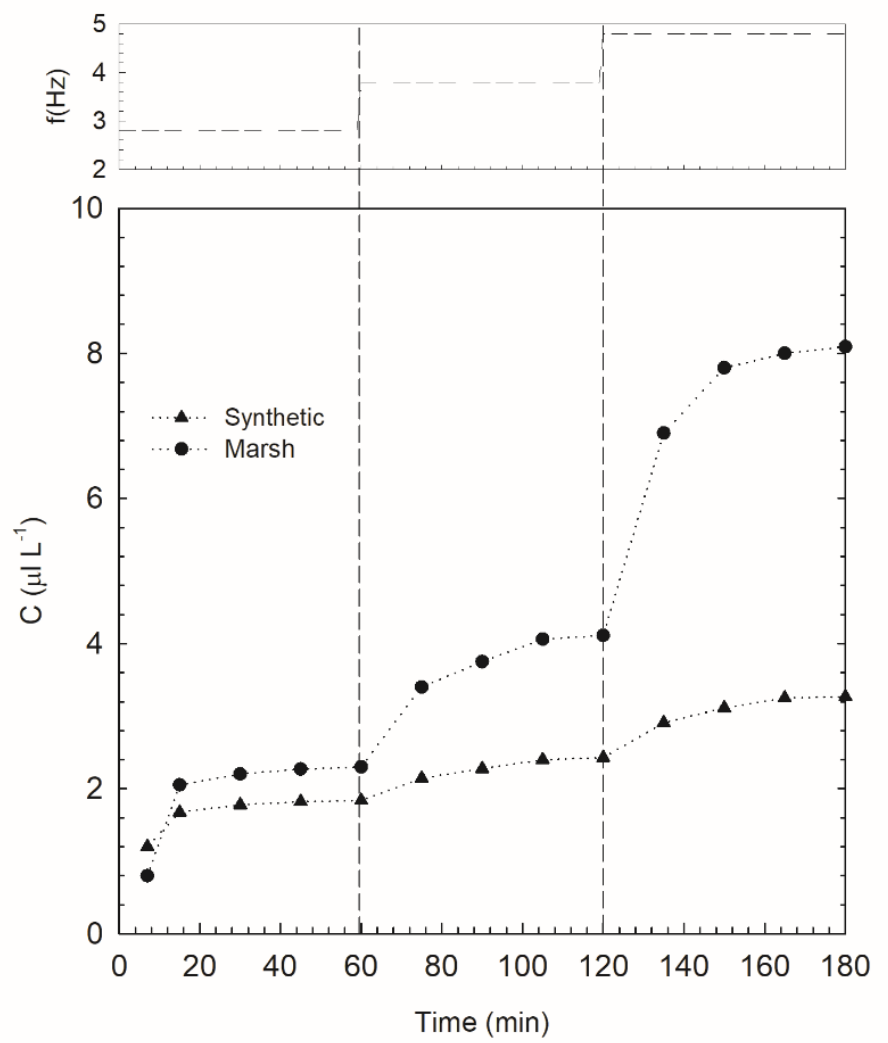

Figure 7. Time evolution of the sediment concentration for experiments carried out for rigid vegetation with $\mathrm{SPF}=2.5 \%$, for the synthetic sediment and the marsh sediment. The dashed line at the top panel corresponds to the evolution of the oscillation frequency (f) over the full time period of each experiment run. 


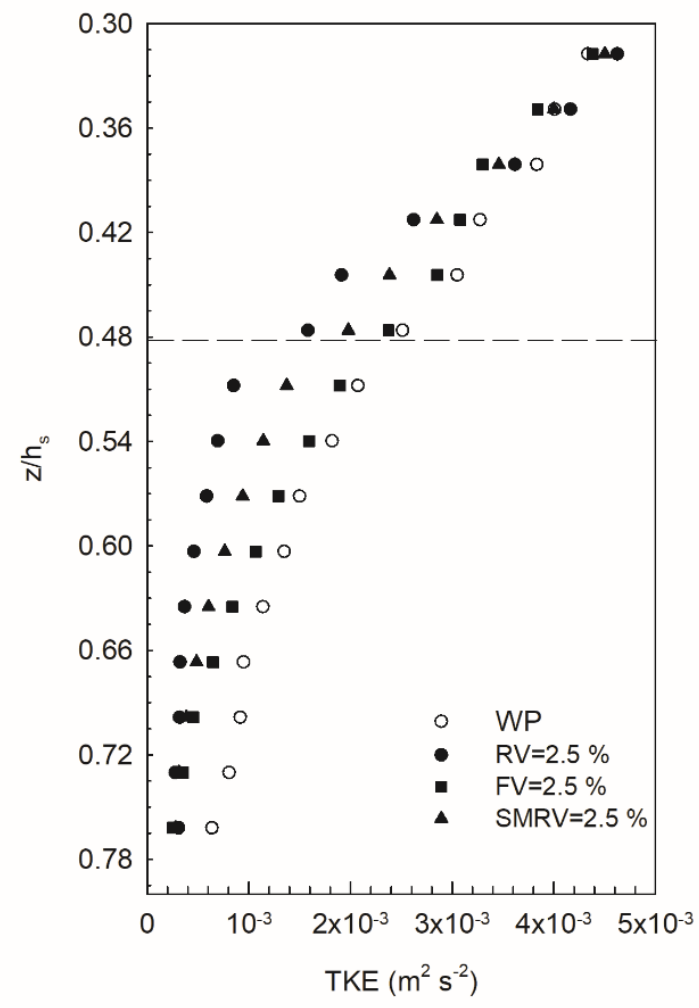

Figure 8. TKE profiles for experimental runs without plants (WP), and with flexible (FV), rigid $(\mathrm{RV})$ and semi rigid vegetation (SMRV), all with $\mathrm{SPF}=2.5 \%$. Grid oscillation frequency was $\mathrm{f}=4.8 \mathrm{~Hz}$ in all cases shown. 

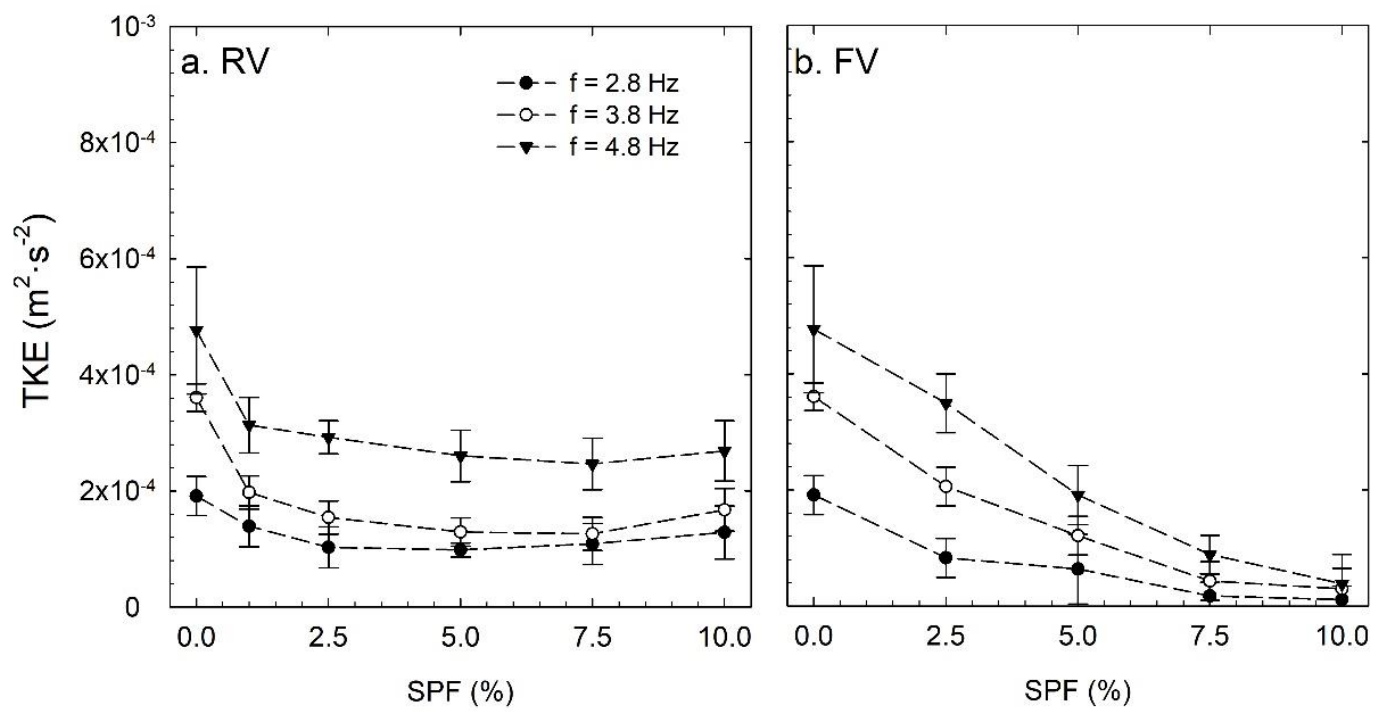

Figure 9. Relationship between the turbulent kinetic energy (TKE) at $z=22 \mathrm{~cm}$ and the solid plant fraction (SPF) of the canopies for different oscillating grid frequencies, $f$, for (a) rigid and (b) flexible canopies. 
a.

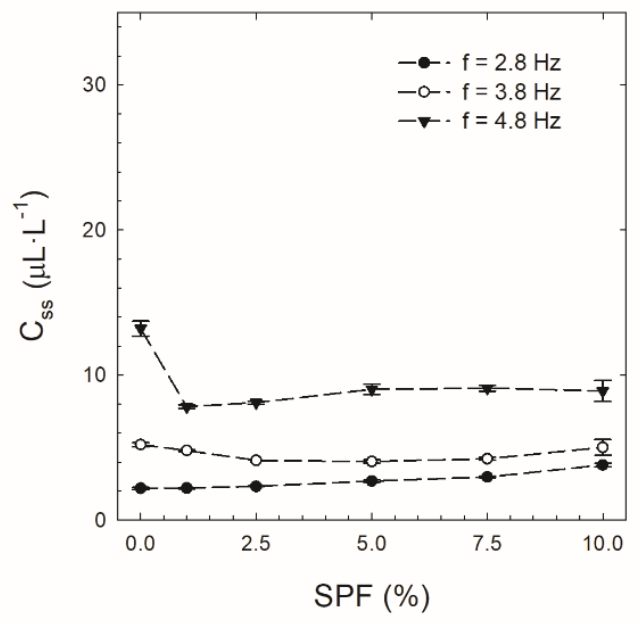

c.

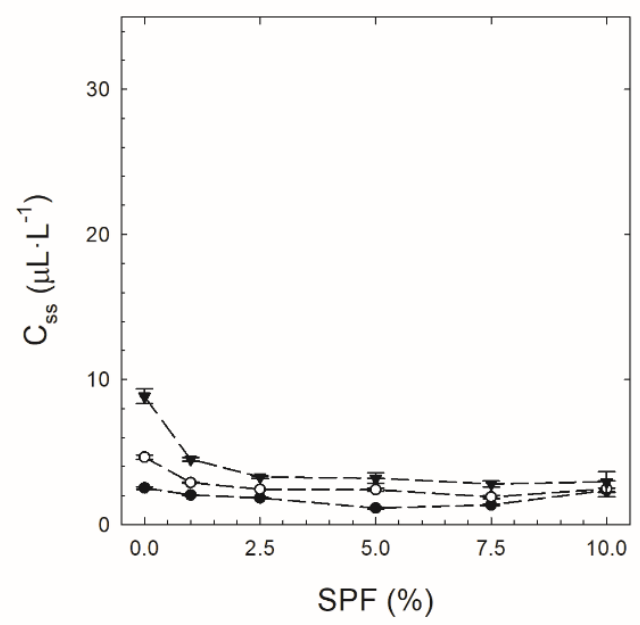

$\mathrm{b}$

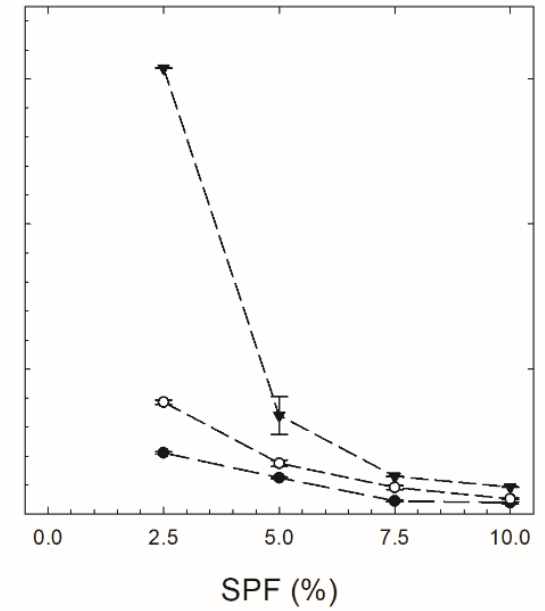

d.

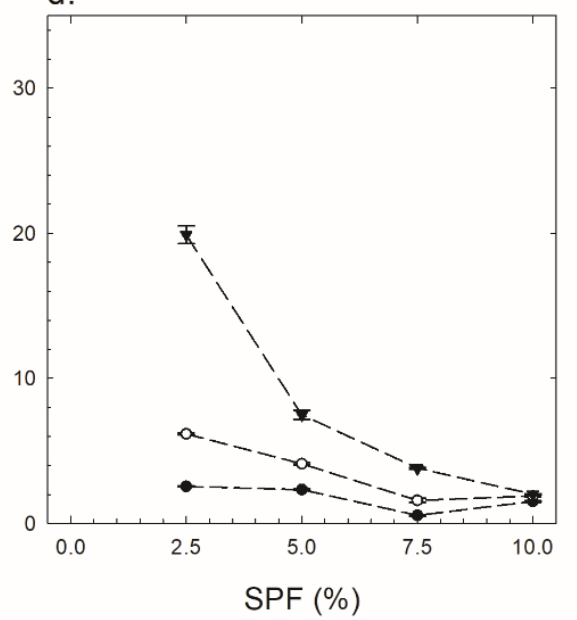

Figure 10. Relationship between the suspended sediment concentration at the steady state $\left(\mathrm{C}_{\mathrm{ss}}\right)$ measured at $\mathrm{z}=0.22 \mathrm{~m}$ and the solid plant fraction (SPF) for different oscillating frequencies (f) for (a and c) rigid, (b and d) flexible canopies, for the marsh (top) and synthetic sediment (bottom). 


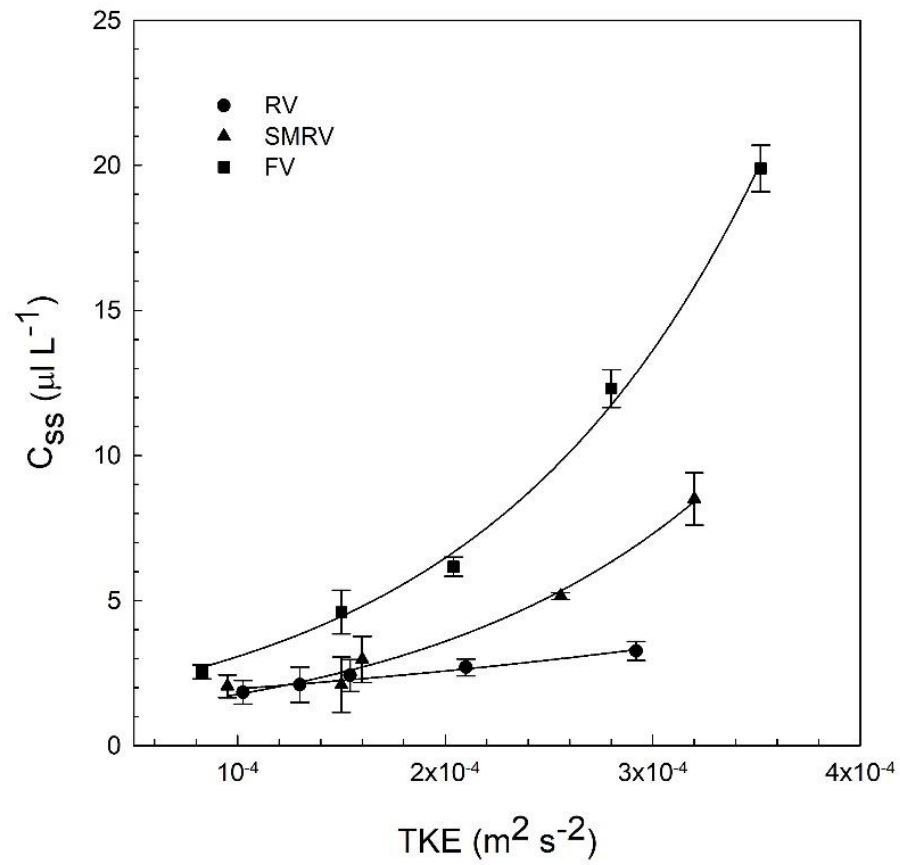

Figure 11. Dependence of the sediment concentration on the suspension at $z=22 \mathrm{~cm}$ (i.e. $z / h_{s}=0.7$ ) and the turbulent kinetic energy, for the three types of canopies (rigid, semi-rigid and flexible) for a solid plant fraction of $2.5 \%$. For all runs, a two-day synthetic consolidated bed was used. Vertical error bars are calculated from the standard deviation of different measurements of the same run. Solid lines represent the exponential best fit curve through the data obtained in each case. The equations of the exponential fitting are $C_{s s}=1.46 \mathrm{e}^{7448 T K E}$ $\left(\mathrm{r}^{2}=0.9968\right)$ for $\mathrm{FV}, C_{S s}=0.87 \mathrm{e}^{7085 T K E}\left(\mathrm{r}^{2}=0.9932\right)$ for $\mathrm{SMRV}$ and $C_{S s}=1.49 \mathrm{e}^{2733 T K E}\left(\mathrm{r}^{2}=0.9622\right)$ for RV. 


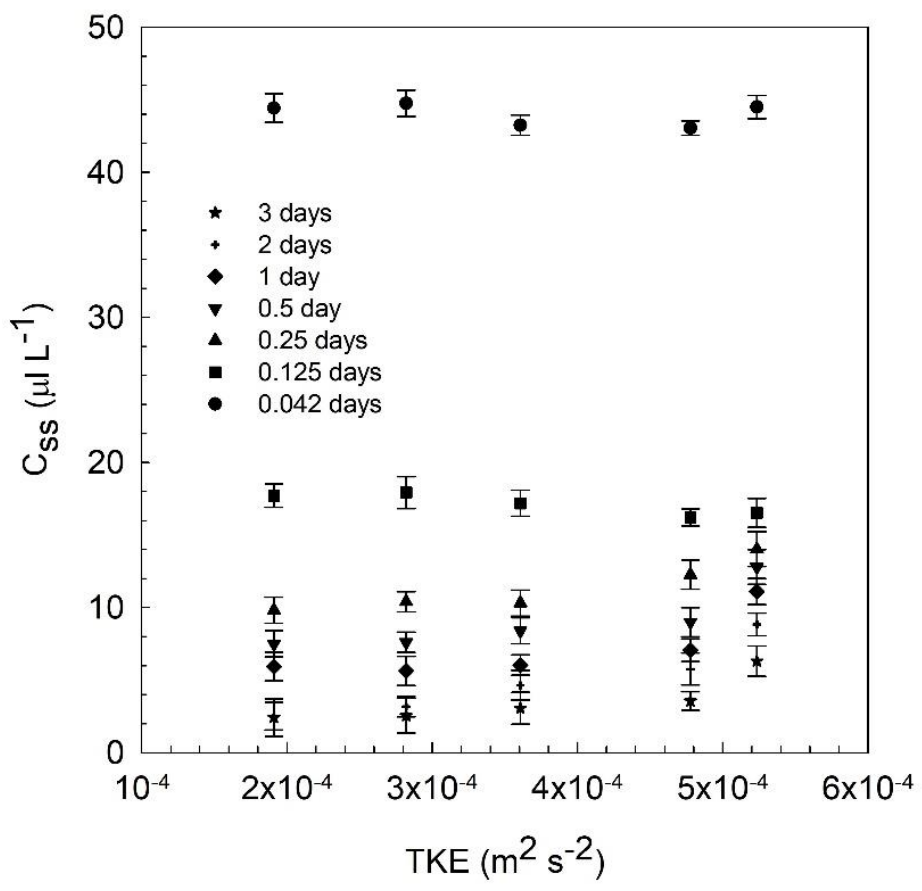

Figure 12. Relationship between the sediment concentration of the suspension at $z=22 \mathrm{~cm}$ (i.e. $z / h_{s}=0.7$ ) and the turbulent kinetic energy, for the seven bed consolidation times, varying from one hour to three days. For all runs, the synthetic type sediment was used. Vertical error bars are calculated from the standard deviation of different measurements of the same run. 


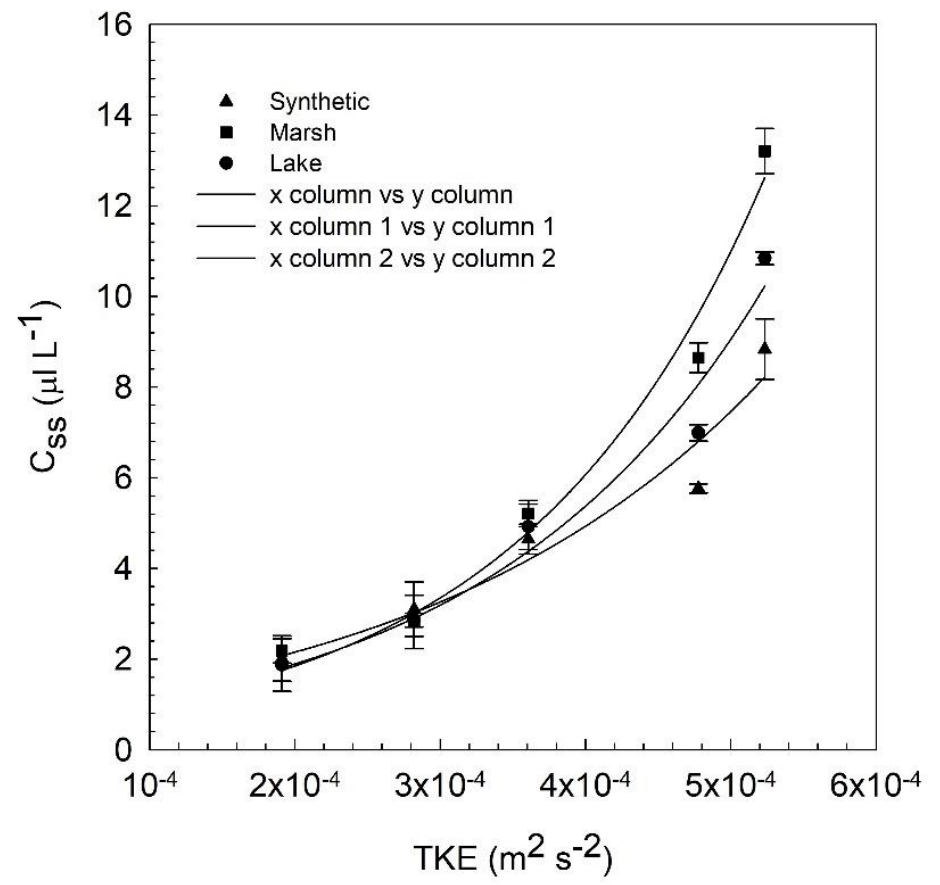

Figure 13. Relationship between the sediment concentration $C_{s s}$ at $z=22 \mathrm{~cm}$ at the steady state and the turbulent kinetic energy, for the three types of sediments (synthetic, lake and marsh) for the without-plants experiments. For all runs, a two-day consolidated bed was used. Vertical error bars are calculated from the standard deviation of different measurements of the same run. Solid lines represent the exponential best fit curve through the data obtained in each case. The equations of the exponential fitting are $C_{s S}=0.56 \mathrm{e}^{5937 T K E}\left(\mathrm{r}^{2}=0.9798\right)$ for the marsh sediment, $C_{s s}=0.67 \mathrm{e}^{5213 T K E}\left(\mathrm{r}^{2}=0.9644\right)$ for the lake sediment and $C_{S s}=0.94 \mathrm{e}^{4139 T K E}\left(\mathrm{r}^{2}=0.9398\right)$ for the synthetic sediment. 


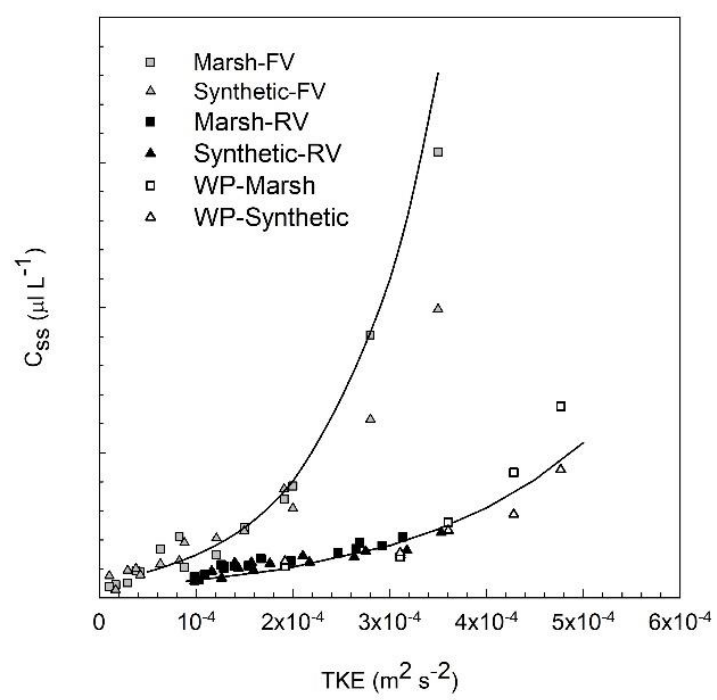

Figure 14. Relationship between the sediment concentration of the suspension at $\mathrm{z}=22 \mathrm{~cm}$ and the turbulent kinetic energy, for the (a) rigid vegetation runs and no vegetation runs, and (b) flexible vegetation, for both the synthetic and marsh sediment. For all runs a two-day consolidated bed was used. Solid lines represent the exponential best fit curve through the obtained data in each case. The equations of the exponential fitting are $\mathrm{C}_{\mathrm{ss}}=1.04 \mathrm{e}^{4063 \mathrm{TKE}}$ $\left(\mathrm{r}^{2}=0.9076\right)$ for $\mathrm{RV}$, and $\mathrm{C}_{\mathrm{ss}}=1.11 \mathrm{e}^{9257 \mathrm{TKE}}\left(\mathrm{r}^{2}=0.9070\right)$ for $\mathrm{FV}$. 